\title{
Searching for pseudo Nambu-Goldstone boson dark matter production in association with top quarks
}

\author{
Ulrich Haisch, ${ }^{a}$ Giacomo Polesello ${ }^{b}$ and Stefan Schulte ${ }^{a, c}$ \\ ${ }^{a}$ Max Planck Institute for Physics, \\ Föhringer Ring 6, 80805 München, Germany \\ ${ }^{b}$ INFN, Sezione di Pavia, \\ Via Bassi 6, 27100 Pavia, Italy \\ ${ }^{c}$ Physik-Department, Technische Universität München, \\ 85748 Garching, Germany \\ E-mail: haisch@mpp.mpg.de, giacomo.polesello@cern.ch, \\ sschulte@mpp.mpg.de
}

AbStract: Pseudo Nambu-Goldstone bosons (pNGBs) are attractive dark matter (DM) candidates, since they couple to the Standard Model (SM) predominantly through derivative interactions. Thereby they naturally evade the strong existing limits inferred from DM direct detection experiments. Working in an effective field theory that includes both derivative and non-derivative DM-SM operators, we perform a detailed phenomenological study of the Large Hadron Collider reach for pNGB DM production in association with top quarks. Drawing on motivated benchmark scenarios as examples, we compare our results to other collider limits as well as the constraints imposed by DM (in)direct detection experiments and the relic abundance. We furthermore explore implications on the viable parameter space of pNGB DM. In particular, we demonstrate that DM direct detection experiments become sensitive to many pNGB DM realisations once loop-induced interactions are taken into account. The search strategies and pNGB DM benchmark models that we discuss can serve as a starting point for dedicated experimental analyses by the ATLAS and the CMS collaborations.

Keywords: Beyond Standard Model, Higgs Physics

ArXiv EPrint: 2107.12389 


\section{Contents}

1 Introduction 1

2 Theoretical framework 3

3 MC generation and detector simulation $\quad 4$

4 Mono- $X$ analysis strategies $\quad 6$

$4.1 t X+E_{T}^{\text {miss }}$ final states $\quad 6$

$4.2 j+E_{T}^{\text {miss }}$ final state 9

5 Constraints from $t X+E_{T}^{\text {miss }}$ and $j+E_{T}^{\text {miss }}$ searches at the LHC $\quad 10$

6 Constraints from invisible Higgs decays at the LHC 11

7 Constraints from DM (in)direct detection and the relic density 13

8 Discussion 16

$\begin{array}{ll}\text { A Supplementary material } & 21\end{array}$

\section{Introduction}

Weakly interacting massive particles (WIMPs) have been the prime dark matter (DM) candidate for more than three decades because they can give rise to the correct abundance of DM today via thermal freeze-out production. However, the null results from DM direct and indirect detection experiments (see for instance [1, 2]) along with the failure to observe anomalous missing transverse energy $\left(E_{T}^{\text {miss }}\right)$ production at the Large Hadron Collider (LHC) (see [3] for an experimental status report) have by now ruled out large portions of the parameter space of the simplest WIMP hypotheses such as the neutralino in supersymmetric theories.

Compelling examples of still viable WIMP models are provided by scenarios in which DM consists of composite pseudo Nambu-Goldstone bosons (pNGBs). Models of this type can address simultaneously the electroweak (EW) hierarchy problem of the Standard Model (SM) and the DM puzzle [4], and as a result have received notable attention in recent years [5-32]. In models in which both the SM Higgs boson and DM emerge from a TeV-scale strongly-coupled sector as pNGBs, one key feature is that the leading coupling between the SM and DM is provided by higher-dimensional, derivative interactions with the Higgs field. The derivative Higgs portal mediates $s$-wave annihilation to SM particles, but leads to a strong suppression of the DM scattering rate on ordinary matter. Thermal 
freeze-out can therefore yield the observed relic density for a DM mass of the order of $100 \mathrm{GeV}$, while the current severe limits of DM direct detection experiments are naturally evaded. Probes of composite pNGB DM include indirect detection searches and collider experiments. The collider reach on the derivative Higgs portal has been recently analysed in vector-boson-fusion (VBF) Higgs production [25], finding a limited sensitivity at the LHC. This motivates studies of the indirect constraints on the derivative Higgs portal that arise from off-shell single-Higgs and on-shell double-Higgs production at hadron colliders [33, 34].

Besides the derivative Higgs portal, composite pNGB DM models necessarily contain additional interactions to provide a potential and Yukawa couplings for the Higgs boson and a mass for the DM candidate. A theoretically motivated situation is one in which DM couples most strongly to the third generation of SM fermions. At the level of dimensionsix operators, such interactions can either be of Yukawa type or involve the product of a DM and a SM current. Detailed studies of the DM phenomenology of composite pNGB models where the Goldstone shift symmetry of DM is broken by the top or the bottom Yukawa coupling can be found in $[16,20]$. These analyses show that scenarios in which the shift symmetry is broken in the bottom sector are significantly less constrained by DM direct detection than those in which the top sector provides the leading symmetry breaking. In composite pNGB models with sizeable DM-SM Yukawa couplings and a successful DM phenomenology, the leading $E_{T}^{\text {miss }}$ signature is therefore expected to be DM production in association with bottom quarks. Unfortunately, this process can only be constrained poorly at the LHC [35-37]. If, on the other hand, effective current-current interactions provide a relevant portal between the dark and the visible sector, large DM-top couplings are compatible with both the bounds from DM (in)direct detection and the observed relic abundance if DM is sufficiently heavy [25]. As a result, such composite pNGB DM models can be tested at the LHC by searching for DM production in association with top-quark pairs $\left(t \bar{t}+E_{T}^{\text {miss }}\right)$ or a top quark and a $W$ boson $\left(t W+E_{T}^{\text {miss }}\right)$. These mono- $X$ channels, from now on referred to as $t X+E_{T}^{\text {miss }}$, have received a lot of attention from the DM collider community [35, 36, 38-48].

The main goal of this article is to analyse the LHC reach of the $t X+E_{T}^{\text {miss }}$ channels and to constrain the parameter space of composite pNGB DM models. To keep our discussion as model-independent as possible we will work in an effective field theory focusing on the subset of operators that lead to DM production in association with top quarks. Through loops such operators also lead to a $j+E_{T}^{\text {miss }}$ signal, and we study the limits on the parameter space of the pNGB DM effective field theory that are imposed by the corresponding monojet searches. We then offer a comprehensive discussion of the phenomenological features of pNGB DM models, including an analysis of the DM direct and indirect detection constraints as well as of the physics of thermal freeze-out. The search strategies and pNGB DM benchmark models that we discuss are meant to set the stage for dedicated experimental analyses by ATLAS and CMS.

Our work is organised as follows. In section 2 we describe the structure of the composite pNGB DM models that we consider. Our Monte Carlo (MC) generation and our detector simulation are spelled out in section 3 , while section 4 describes the analysis strategies to search for the relevant mono- $X$ signals. In section 5 we examine the sensitivity of the 
studied pNGB DM signatures at upcoming LHC runs. The present and future constraints on the pNGB DM effective field theory that arise from invisible Higgs decays are discussed in section 6 . The relevant non-collider limits are presented in section 7 . We discuss our main results and give an outlook in section 8. The impact of the assumed systematic background uncertainties on our $t X+E_{T}^{\text {miss }}$ projections is studied in the supplementary material that can be found in appendix A.

\section{Theoretical framework}

Throughout this article we will consider theories in which both the SM Higgs doublet $H$ and the DM candidate $\chi$ arise as light pNGBs from a strongly-coupled sector. The DM candidate is a singlet under the SM gauge group and we assume it to be a complex scalar. The terms of the interaction Lagrangian relevant for the further discussion can be written as [25]

$$
\begin{aligned}
\mathcal{L}_{\chi H} & =\frac{c_{d}}{f^{2}} \partial_{\mu}|\chi|^{2} \partial^{\mu}|H|^{2}-\lambda|\chi|^{2}|H|^{2} \\
\mathcal{L}_{\chi \psi} & =\frac{|\chi|^{2}}{f^{2}}\left(c_{t} y_{t} \bar{q}_{L} \tilde{H} t_{R}+\text { h.c. }\right)+\frac{i}{f^{2}} \chi^{*} \stackrel{\leftrightarrow}{\partial_{\mu}} \chi \sum_{\psi=q_{L}, t_{R}, b_{R}} d_{\psi} \bar{\psi} \gamma^{\mu} \psi .
\end{aligned}
$$

Here the terms in $\mathcal{L}_{\chi H}$ correspond to the derivative and marginal Higgs portal, respectively, while the terms in $\mathcal{L}_{\chi \psi}$ correspond to the Yukawa-type DM-top coupling and the currentcurrent type interactions between DM and the third-generation SM quarks, respectively. The common decay constant of the pNGBs is denoted by $f$, while the coefficients $c_{i}, \lambda$ and $d_{j}$ are $O(1)$ constants that we assume to be real such that CP is conserved. In (2.1) we have furthermore used the definition $\chi^{*} \stackrel{\leftrightarrow}{\partial_{\mu}} \chi=\chi^{*} \partial_{\mu} \chi-\chi \partial_{\mu} \chi^{*}$, and $q_{L}=\left(t_{L}, b_{L}\right)^{T}$ denotes the left-handed third-generation quark doublet, $t_{R}\left(b_{R}\right)$ is the right-handed top-quark (bottomquark) singlet, $y_{t}=\sqrt{2} m_{t} / v$ is the top Yukawa coupling with $m_{t} \simeq 163 \mathrm{GeV}$ the top mass and $v \simeq 246 \mathrm{GeV}$ the Higgs vacuum expectation value (VEV), and we have defined $\tilde{H}^{i}=\varepsilon_{i j}\left(H^{j}\right)^{*}$ with $\varepsilon_{i j}$ totally antisymmetric and $\varepsilon_{12}=1$. Notice that the current-current type operator in $\mathcal{L}_{\chi \psi}$ is absent if hidden-charge conjugation (i.e. $\chi \rightarrow-\chi^{*}$ and $\psi \rightarrow \psi$ ) is preserved as in all explicit pNGB DM models studied in [20]. Moreover, this operator vanishes trivially if the DM candidate is a real scalar.

Besides the four types of interactions introduced in (2.1), the full pNGB DM effective field theory can contain additional dimension-six operators such as $\chi^{*} \stackrel{\leftrightarrow}{\partial_{\mu}} \chi \partial_{\nu} B^{\mu \nu}$ and $|\chi|^{2} V_{\mu \nu} V^{\mu \nu}$. Here $V_{\mu \nu}=B_{\mu \nu}, W_{\mu \nu}^{i}, G_{\mu \nu}^{a}$ denotes the $\mathrm{U}(1)_{Y}, \mathrm{SU}(2)_{L}$ and $\mathrm{SU}(3)_{C}$ fieldstrength tensor, respectively. Since the latter two types of operators do not lead to a relevant $t X+E_{T}^{\text {miss }}$ signal at tree level, such terms are not directly testable in DM production in association with top quarks. In contrast, the presence of DM couplings with gauge bosons may have an important impact on the calculation of the DM (in)direct detection bounds and on the derivation of the DM relic density. To highlight the complementarity of collider and non-collider bounds in a simple fashion, we therefore restrict our analysis to the subclass of models in which the leading effects at the scale at which DM and the Higgs boson emerge as composite pNGBs are well captured by the effective Lagrangians $\mathcal{L}_{\chi H}$ 
and $\mathcal{L}_{\chi \psi}$. However, we will discuss and include pNGB DM interactions with gauge bosons that are generated from (2.1) once radiative corrections are included, whenever these yield significant contributions (see section 7).

We finally mention that under the assumption that the cancellation of gauge anomalies only depends on the SM fermion representations and not on the structure of the pNGB DM effective field theory (in particular the coefficients $d_{\psi}$ in (2.1)), the current-current type DM-top operator does not lead to a $j+E_{T}^{\text {miss }}$ signal. In practice this requires one to introduce local counterterms that cancel the anomalous contributions in the five-point diagrams like the one shown on the right-hand side in figure 2 - see [49-51] for related discussions of gauge anomalies in the context of the so-called SMEFT. Since we envisage that (2.1) describes new-physics scenarios in which the full SM gauge symmetry is preserved, a matching calculation in the full theory will always result in the required anomaly cancellation, and consequently a cancellation of the current-current type contributions to the mono-jet signature for any value of the parameters $d_{\psi}$.

\section{MC generation and detector simulation}

In our work we study the $t \bar{t}+E_{T}^{\text {miss }}$, the $t W+E_{T}^{\text {miss }}$ and the $j+E_{T}^{\text {miss }}$ signatures that arise from insertions of the pNGB DM operators introduced in (2.1). Examples of leadingorder (LO) diagrams that involve DM-Higgs and DM-top operators are displayed in figure 1 and figure 2, respectively. Notice that only DM-top operators can lead to a LO mono-jet signal as illustrated by the graph shown on the right-hand side in figure 2. All our signal predictions assume proton-proton $(p p)$ collisions at a centre-of-mass (CM) energy of $14 \mathrm{TeV}$ and are calculated using a FeynRules 2 [52] implementation of the Lagrangian (2.1) in the UFO format [53]. The generation and showering of the mono- $X$ samples is performed with MadGraph5_aMC@NLO [54] at LO and PYTHIA 8.2 [55], respectively, using NNPDF3. 0 parton distribution functions (PDFs) [56]. In order to preserve both spin correlations and finitewidth effects, final-state top quarks and $W$ bosons are decayed with MadSpin [57].

In the case of the $t X+E_{T}^{\text {miss }}$ signatures, all SM processes that contain at least two charged leptons $(\ell=e, \mu)$ coming from the decay of an EW gauge boson $V=W, Z$ are included in the background simulation. We do not consider backgrounds with either fake electrons from jet misidentification or with real non-isolated leptons from the decay of heavy-flavoured hadrons. A reliable estimate of these backgrounds depends on a detailed simulation of detector effects beyond the scope of this article. For the most recent ATLAS analyses involving leptonic final states [47, 48], the background from non-prompt leptons is a few percent of the total background. The backgrounds from $t \bar{t}[58], t W[59], W W$, $W Z$ and $Z Z$ production $[60,61]$ are all generated at the next-to-leading order (NLO) in QCD with POWHEG BOX [62]. The $V+$ jets backgrounds are generated at LO using MadGraph5_aMC@NLO and include up to four additional jets. MadGraph5_aMC@NLO is also used to simulate the $t \bar{t} V$ backgrounds with a multiplicity of up to two jets, while the $t Z$ and $t W Z$ backgrounds are obtained at LO with the same MC generator. All partonic events are showered with PYTHIA 8.2. The samples produced with POWHEG BOX are normalised to the corresponding NLO QCD cross sections, except for $t \bar{t}$, which is normalised to the 

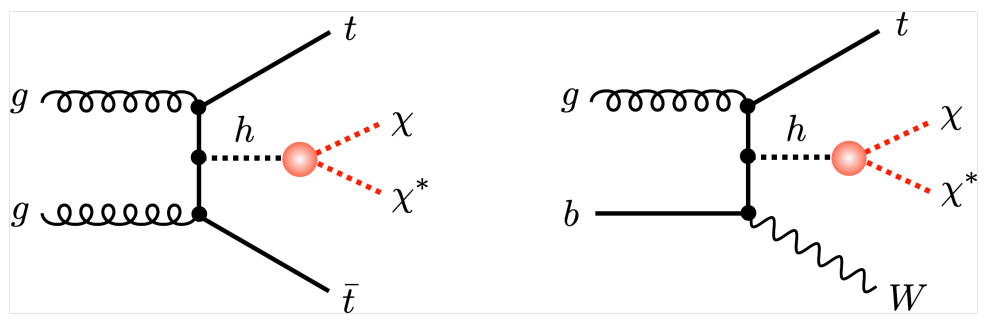

Figure 1. Examples of diagrams with insertions of the DM-Higgs operators (filled red circles) in (2.1) that lead to a $t \bar{t}+E_{T}^{\text {miss }}$ (left) and $t W+E_{T}^{\text {miss }}$ (right) signal. The black dots indicate SM interactions.
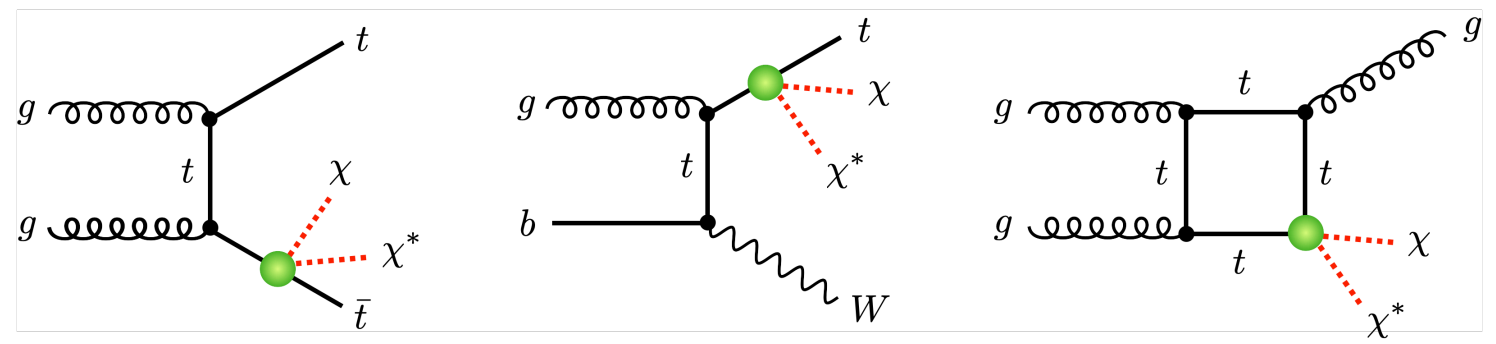

Figure 2. Assortment of graphs with insertions of the DM-top operators (filled green circles) entering (2.1) that give rise to a $t \bar{t}+E_{T}^{\text {miss }}$ (left), $t W+E_{T}^{\text {miss }}$ (middle) and $j+E_{T}^{\text {miss }}$ (right) signature.

cross section obtained at the next-to-next-to-leading order (NNLO) in QCD plus next-tonext-to-leading logarithmic QCD corrections [63, 64]. The $V+$ jets samples are normalised to the NNLO QCD cross sections [65, 66] and the $t \bar{t} V$ samples are normalised to the NLO QCD cross section as calculated by MadGraph5_aMC@NLO.

For the $j+E_{T}^{\text {miss }}$ signature, the dominant SM backgrounds arise from $V+$ jets production. The only relevant process not included in the $t X+E_{T}^{\text {miss }}$ backgrounds described above is the $Z+$ jets channel followed by the decay $Z \rightarrow \nu \bar{\nu}$. Like in the earlier works $[67,68]$ the corresponding background is generated at LO with MadGraph5_aMC@NLO, and can contain up to two additional jets. The generation is performed in slices of the vector-boson transverse momentum $\left(p_{T}\right)$, and the resulting events are showered with PYTHIA 8.2 employing a Catani-Krauss-Kuhn-Webber jet matching procedure [69]. The inclusive signal region IM3 of the ATLAS analysis [70] requires $E_{T}^{\text {miss }}>350 \mathrm{GeV}$, and for these selections the background from $V+$ jets production amounts to around $95 \%$ of the total SM background. The $V+$ jets samples are normalised such that the different contributions match the number of events in the IM3 signal region as estimated by ATLAS scaled from a CM energy of $13 \mathrm{TeV}$ to $14 \mathrm{TeV}$ and to the appropriate integrated luminosity. The additional minor backgrounds from $t \bar{t}, t W$ and diboson production are the same as in the $t X+E_{T}^{\text {miss }}$ case.

The actual physics analyses use experimentally identified electrons, muons, photons, jets $(j)$ and $E_{T}^{\text {miss }}$. These objects are constructed from the stable particles in the generator output. Jets are built out of the momenta of all the stable particles depositing energy in the calorimeter except for muons using the anti- $k_{t}$ algorithm [71] with a radius parameter 
of $R=0.4$, as implemented in FastJet [72]. Jets originating from the hadronisation of bottom quarks ( $b$-jets) are experimentally identified (i.e. $b$-tagged) with high efficiency. The $\vec{p}_{T}^{\text {miss }}$ vector with magnitude $E_{T}^{\text {miss }}$ is constructed from the transverse momenta of all the invisible particles in the event. Detector effects are simulated by smearing the momenta of the analysis objects and by applying efficiency factors where applicable. The used smearing and efficiency functions are tuned to reproduce the performance of the ATLAS detector $[73,74]$. In particular, the performance of the ATLAS $b$-tagging algorithm is taken from [75]. For the mono- $X$ analyses performed in this article, a $b$-tagging working point is chosen that yields a $b$-tagging efficiency of $77 \%$, a $c$-jet rejection of 5 and a light-flavour jet rejection of 110 . More details on our detector simulation can be found in the earlier papers $[42,76]$.

\section{Mono- $X$ analysis strategies}

Below we describe the analysis strategies to target the $t X+E_{T}^{\text {miss }}$ and $j+E_{T}^{\text {miss }}$ signals that are due to the interactions described by (2.1). For each analysis strategy we define the signal regions, spell out all selection criteria and quantify the systematic uncertainties that plague the search strategy in question.

\section{1 $t X+E_{T}^{\text {miss }}$ final states}

The considered signal events include the decays of two $W$ bosons. We address the final states where only one or both of the $W$ bosons decay into charged leptons, which hereafter will be called semileptonic or fully-leptonic, respectively. Our $t X+E_{T}^{\text {miss }}$ analysis is based on the definition of three orthogonal signal regions. The first two signal regions target the associated production of a $t \bar{t}$ pair and DM with SR1 (SR2) selecting semileptonic (fully-leptonic) events. The third signal region called SR3 instead considers the associated production of a top quark, a $W$ boson and DM, which is searched for in fully-leptonic events. The corresponding final states therefore involve a single isolated charged lepton and two $b$-tagged jets (SR1), two isolated charged leptons and two $b$-tagged jets (SR2) or two isolated charged leptons and a single $b$-tagged jet (SR3). Notice that $t W+E_{T}^{\text {miss }}$ production typically has a smaller cross section than $t \bar{t}+E_{T}^{\text {miss }}$ production. However, in the case of the two-lepton final state, it has been shown in [45] that it is possible to devise a selection strategy that combines the $t \bar{t}+E_{T}^{\text {miss }}$ and the $t \bar{W}+E_{T}^{\text {miss }}$ channels and has a significantly larger sensitivity than $t \bar{t}+E_{T}^{\text {miss }}$ alone. Such a selection is based on the observation that events produced by a fully-leptonic $t \bar{t}$ decay contain two $\ell b$ pairs for both of which the invariant mass $m_{\ell b}$ is bounded from above by $\sqrt{m_{t}^{2}-M_{W}^{2}} \simeq 153 \mathrm{GeV}$. This is not the case for the $t W$ production which contains only one $\ell b$ pair satisfying this bound. The two processes can thus be separated by defining the variable

$$
m_{b \ell}^{t}=\min \left(\max \left(m_{\ell_{1} j_{a}}, m_{\ell_{2} j_{b}}\right)\right),
$$

and putting a cut on $m_{b \ell}^{t}$ of around $160 \mathrm{GeV}$ to separate $t \bar{t}$ from $t W$ events. In (4.1) the variables $m_{\ell_{1} j_{a}}$ and $m_{\ell_{2} j_{b}}$ denotes the invariant mass of the leading and subleading leptons 


\begin{tabular}{|l|c|}
\hline Variable & SR1 selection \\
\hline$N_{\ell}$ & $=1, \quad p_{T}(\ell)>25 \mathrm{GeV}, \quad|\eta(\ell)|<2.5$ \\
$N_{j}$ & $\geq 4, \quad p_{T}(j)>(80,60,30,25) \mathrm{GeV},|\eta(j)|<2.5$ \\
$N_{b}$ & $\geq 2, \quad p_{T}(b)>(80,25) \mathrm{GeV}, \quad|\eta(b)|<2.5$ \\
\hline$E_{T}^{\text {miss }}$ & $>550 \mathrm{GeV}$ \\
$m_{T}^{\ell}$ & $>180 \mathrm{GeV}$ \\
Topness & $>8$ \\
$m_{\text {top }}^{\text {reclustered }}$ & $>150 \mathrm{GeV}$ \\
$H_{T, \text { sig }}^{\text {miss }}$ & $>15$ \\
$\left|\Delta \phi_{\ell, \text { miss }}\right|$ & $>1.3$ \\
$\left|\Delta \phi_{\text {min }}\right|$ & $>0.9$ \\
$\left|\Delta \phi_{b b}\right|$ & $<2.5$ \\
\hline
\end{tabular}

Table 1. Definition of the signal region SR1. The number of charged leptons, light-flavoured jets and $b$-tagged jets are denoted by $N_{\ell}, N_{j}$ and $N_{b}$, respectively. For further details consult the text.

$\ell_{1}$ and $\ell_{2}$ and the jets $j_{a}$ and $j_{b}$. The minimisation with respect to the jet pairs $j_{a}$ and $j_{b}$ runs over all of the $b$-tagged jets if the number of $b$-tagged jets satisfies $N_{b} \geq 3$ or over the $b$-tagged jets and the untagged jet with the highest $b$-tagging weight if $N_{b} \leq 2$. Since the three signal regions are designed to have no events in common, the final search sensitivity of the $t X+E_{T}^{\text {miss }}$ channel will be calculated after the statistical combination of SR1, SR2 and SR3. The selection criteria corresponding to the three signal regions are summarised in tables 1 and 2 .

In the case of SR1 the selection requirements are similar to the ones imposed in the signal region DM of [47]. However, some variables have been modified and the values of the cuts have been optimised to our MC simulations of both the signal and the background at the high-luminosity upgrade of the LHC (HL-LHC). The basic selection requires one and only one isolated charged lepton and at least four jets of which exactly two must be tagged as $b$-jets. Furthermore, jets tagged as hadronic decays of a $\tau$ lepton are vetoed. The employed cuts on the $p_{T}$ and pseudorapidities $(\eta)$ of the leptons and jets can be found in table 1. After the initial selections the dominant background is $t \bar{t}$ production with one top quark decaying leptonically and the other one decaying hadronically. This background is strongly reduced by demanding $E_{T}^{\text {miss }}>550 \mathrm{GeV}$ and requiring a lower limit of $180 \mathrm{GeV}$ on the transverse mass of the charged lepton defined as

$$
m_{T}^{\ell}=\sqrt{2\left|\vec{p}_{T}(\ell)\right|\left|\vec{p}_{T}^{\text {miss }}\right|\left(1-\cos \Delta \phi_{\ell, \text { miss }}\right)}
$$

Here $\vec{p}_{T}(\ell)$ denotes the components of the lepton momentum transverse to the beam, $\vec{p}_{T}^{\text {miss }}$ is the vector sum of the transverse momenta of the invisible particles and $\Delta \phi_{\ell, \text { miss }}=$ $\Delta \phi\left(\vec{p}_{T}(\ell), \vec{p}_{T}^{\text {miss }}\right)$ is the azimuthal angular separation between these two vectors. To reject events which are incompatible with top-quark decays, selections on the variables 


\begin{tabular}{|c|c|c|}
\hline Variable & SR2 selection & SR3 selection \\
\hline $\begin{array}{l}N_{\ell} \\
m_{\ell \ell}\end{array}$ & \multirow{2}{*}{\multicolumn{2}{|c|}{$\begin{array}{c}=2, \quad p_{T}(\ell)>(25,20) \mathrm{GeV}, \quad|\eta(\ell)|<2.5 \\
>20 \mathrm{GeV}, \quad Z \text {-boson veto for OS leptons } \\
\geq 1, \quad p_{T}(b)>30 \mathrm{GeV}, \quad|\eta(b)|<2.5\end{array}$}} \\
\hline$N_{b}$ & & \\
\hline$m_{b \ell}^{t}$ & $<160 \mathrm{GeV}$ & $>160 \mathrm{GeV}$ or $N_{j}=1$ \\
\hline$E_{T}^{\mathrm{miss}}$ & $>550 \mathrm{GeV}$ & $>350 \mathrm{GeV}$ \\
\hline$\left|\Delta \phi_{\min }\right|$ & $\mathrm{n} / \mathrm{a}$ & $>0.8$ \\
\hline$\left|\Delta \phi_{\text {boost }}\right|$ & $<1.5$ & $<2.5$ \\
\hline$M_{\text {scal }}$ & $\mathrm{n} / \mathrm{a}$ & $<500 \mathrm{GeV}$ \\
\hline$m_{T 2}$ & $>100 \mathrm{GeV}$, shape fit & $>170 \mathrm{GeV}$ \\
\hline
\end{tabular}

Table 2. As table 1 but for the signal regions SR2 and SR3. More details can be found in the main text.

topness [77] and $m_{\mathrm{top}}^{\text {reclustered }}[47]$ are imposed. An additional rejection of the SM background is achieved with selections on $H_{T, \text { sig }}^{\text {miss }}$, i.e. the ratio of $E_{T}^{\text {miss }}$ built as the vector sum of the momenta of all the signal jets and leptons in the event, reduced by $100 \mathrm{GeV}$ and divided by its experimental resolution [78, 79]. Finally, cuts on the azimuthal angular separations $\Delta \phi_{\ell \text {,miss }}, \Delta \phi_{\text {min }}$ between $\vec{p}_{T}(j)$ and $\vec{p}_{T}^{\text {miss }}$ for the four leading jets and on $\Delta \phi_{b b}$ between the two $b$-tagged jets are imposed as detailed in table 1 .

The basis selection of events is common for the signal regions SR2 and SR3. It consists of the requirement of having exactly two isolated opposite-sign (OS) leptons and the invariant mass of the OS leptons has to fulfil $m_{\ell \ell}>20 \mathrm{GeV}$. If the charged leptons are of the same flavour, events with $71 \mathrm{GeV}<m_{\ell \ell}<111 \mathrm{GeV}$ are discarded to suppress backgrounds where the lepton pair arises from the decay $Z \rightarrow \ell^{+} \ell^{-}$. Furthermore, each event is required to contain at least one $b$-tagged jet. The relevant $p_{T}$ and $\eta$ selections of the OS leptons and $b$-jets are specified in table 2. The first selection that differs between the two signal regions is a cut on the $m_{b \ell}^{t}$ observable defined in (4.1), which for SR2 (SR3) is required to be smaller (larger) than $160 \mathrm{GeV}$. The variable $m_{b \ell}^{t}$ is only defined for events with at least two reconstructed jets and events with only one reconstructed jet are assigned to SR3. Further selections are used to optimise the rejection of the SM backgrounds. In the case of SR2 (SR3) we require $E_{T}^{\text {miss }}>550 \mathrm{GeV}\left(E_{T}^{\text {miss }}>350 \mathrm{GeV}\right)$. The four leading jets furthermore have to satisfy $\left|\Delta \phi_{\min }\right|>0.8$ in the signal region SR3. The variable $\Delta \phi_{\text {boost }}$ defined as the azimuthal angle difference between $\vec{p}_{T}^{\text {miss }}$ and the vector sum of $\vec{p}_{T}^{\text {miss }}, \vec{p}_{T}\left(\ell_{1}\right)$ and $\vec{p}_{T}\left(\ell_{2}\right)$, must satisfy the requirement $\left|\Delta \phi_{\text {boost }}\right|<1.5\left(\left|\Delta \phi_{\text {boost }}\right|<2.5\right)$ for SR2 (SR3). In the case of the signal region SR3, we additionally demand that the scalar sum $M_{\text {scal }}$ of the transverse momenta of all the jets observed in the event satisfies $M_{\text {scal }}<500 \mathrm{GeV}$. Finally, in the signal region SR2 we require $m_{T 2}>100 \mathrm{GeV}$ and fit the shape of the $m_{T 2}$ distribution (see for instance [45]), whereas for the signal region SR3 we impose the cut $m_{T 2}>170 \mathrm{GeV}$. Here $m_{T 2}$ denotes the stransverse mass introduced in [80]. 
Assuming an integrated luminosity of $3 \mathrm{ab}^{-1}$ at a CM energy of $14 \mathrm{TeV}$, the number of background events surviving the discussed requirements amounts to 123, 34 and 48 in the case of SR1, SR2 and SR3, respectively. The signal efficiency depends on the DM mass and on the specific pNGB DM model, and in the considered cases it is between a few tens of a percent and a few percent. Given the relatively large number of surviving background events, the experimental reach will depend sensitively on the systematic uncertainty of the estimated SM backgrounds. The size of these uncertainties depends on the detector performance and the techniques used for the background evaluation, which are typically based on a mixed MC and data-driven approach. Existing LHC analyses addressing signatures and a phase space similar to our $t X+E_{T}^{\text {miss }}$ strategy have background uncertainties of $10 \%$ to $30 \%$ (see $[36,47,48]$ ). In our numerical analysis we will assume a $15 \%$ uncertainty on the backgrounds and a $5 \%$ uncertainty on the pNGB DM signals. The latter uncertainty should account for the effect of scale variations and PDF uncertainties on the signal modelling.

In addition to the analysis strategy described in detail above, we have also studied

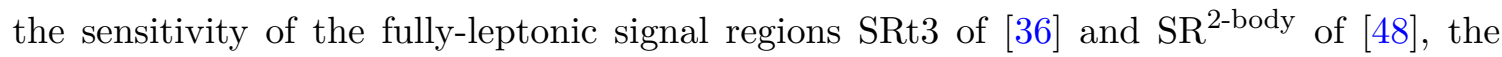
semileptonic signal region DM of [47] and the fully-hadronic signal regions SRt1 and SRt2 of [36] and SRA-TT of [81] to the parameter space of the pNGB DM effective field theory. Our analyses rely in these cases on CheckMATE 2 [82], which uses DELPHES 3 [83] as a fast detector simulation. We find that for what concerns leptonic final states, the best limits on the parameters of (2.1) follow either from the signal region DM or $\mathrm{SR}^{2 \text {-body }}$, while in the case of a fully-hadronic search the strategies SRt2 and SRA-TT fare equally well. It furthermore turns out that the event selections employed in $[36,47,48,81]$ perform at most as good but not better than our optimised $t X+E_{T}^{\text {miss }}$ search strategy. We finally observe that for comparable sets of selection criteria the results from our parametrised simulation and the recast of the ATLAS analyses are in good agreement which validates our simulation approach.

\section{$4.2 j+E_{T}^{\text {miss }}$ final state}

In the case of the $j+E_{T}^{\text {miss }}$ final state, the relevant pNGB DM signal consists of a single high-transverse momentum jet and $E_{T}^{\text {miss }}$ associated to the production of a pair of DM particles. The signature therefore resembles the canonical mono-jet signal, which has received a significant amount of experimental [84-87] and theoretical [88] attention at the LHC, resulting in high-precision estimates of the dominant $E_{T}^{\text {miss }}$ backgrounds that are associated to the production of an EW gauge boson accompanied by at least one high-transverse momentum jet.

In our article we rely on the latest ATLAS mono-jet analysis [70]. Specifically, we employ $E_{T}^{\text {miss }}>350 \mathrm{GeV}$ and require a high-transverse momentum jet with $p_{T}(j)>150 \mathrm{GeV}$ within $|\eta(j)|<2.4$, and no more than four jets with $p_{T}(j)>30 \mathrm{GeV}$ within $|\eta(j)|<2.8$. The selection $\left|\Delta \phi_{\min }\right|>0.4$ is used to fully suppress the multi-jet background. All events containing a reconstructed electron or muon, or the hadronic decay of a tau lepton are rejected. Our selection thus closely resembles the signal region IM3 of [70]. The systematic uncertainty quoted by ATLAS in IM3 is 1.4\%, and we adopt this value as the systematic uncertainty on the total number of background events. Since we perform a multi-bin com- 
parison of the shape of the $E_{T}^{\text {miss }}$ variable, we also need to take into account uncertainties related to the $E_{T}^{\text {miss }}$ shape. For each of the $E_{T}^{\text {miss }}$ bins considered in the analysis, ATLAS gives an uncertainty which increases from around $1.4 \%$ to $4 \%$ between $350 \mathrm{GeV}$ to $1.2 \mathrm{TeV}$. We apply these systematic uncertainties as bin-by-bin shape uncertainties in our $j+E_{T}^{\text {miss }}$ analysis. For the bins between $1.5 \mathrm{TeV}$ and $2 \mathrm{TeV}$ we furthermore assume an uncertainty of $5 \%$, while we take an uncertainty of $8 \%$ for the total number of events in the overflow bin with $E_{T}^{\text {miss }}>2 \mathrm{TeV}$. Notice that our uncertainty treatment corresponds to taking the uncertainties among different $E_{T}^{\text {miss }}$ bins to be uncorrelated. In addition, since the statistical uncertainties of the control regions, that are used to constrain the background, will get reduced with more luminosity, also the systematic uncertainties are expected to decrease with larger data samples. We thus believe that our mono-jet study provides conservative results when applied to the full data set of the HL-LHC.

\section{Constraints from $t X+E_{T}^{\text {miss }}$ and $j+E_{T}^{\text {miss }}$ searches at the LHC}

On the basis of the selection criteria given in section 4, we will study the LHC sensitivity to the discussed mono- $X$ signatures. For each signature and each studied pNGB DM benchmark, we evaluate the value of the cross section which can be excluded at $95 \%$ confidence level (CL) normalised to the nominal LO cross section for the relevant model realisation as calculated by MadGraph5_aMC@NLO. The experimental sensitivity is evaluated using a test statistic based on a profiled likelihood ratio and we make use of the CLs method [91] as implemented in RooStats [92].

In table 3 we present the $95 \%$ CL bounds that derive from our $t X+E_{T}^{\text {miss }}$ analysis for seven different DM masses in the range from $70 \mathrm{GeV}$ to $1 \mathrm{TeV}$. DM masses $m_{\chi}<m_{h} / 2$ where $m_{h} \simeq 125 \mathrm{GeV}$ is the SM Higgs mass are not considered, because in this case invisible Higgs decays generically represent the best way to probe pNGB DM (see the discussion in section 6). The shown limits correspond to the full data set of $3 \mathrm{ab}^{-1}$ that the HL-LHC is expected to collect at a CM energy of $14 \mathrm{TeV}$. Only one free pNGB DM effective field theory parameter is allowed at a time. One observes that HL-LHC $t X+E_{T}^{\text {miss }}$ searches are most sensitive to the current-current type DM-fermion operators followed by the derivative Higgs portal operator and the Yukawa-type DM-top operator. The most difficult operator to probe is the marginal Higgs portal, since it leads compared to the other pNGB DM effective field theory interactions in (2.1) to softer kinematic distributions, making a background suppression generically harder. Notice that in the case of the marginal Higgs portal we have indicated the limits that correspond to a non-perturbative coupling, i.e. $|\lambda|>4 \pi$, by putting parentheses around the corresponding results. We finally add that for $m_{\chi}=1 \mathrm{TeV}$ the bounds on $f / \sqrt{\left|c_{d}\right|}$ and $f / \sqrt{\left|c_{t}\right|}$ following from our $t X+E_{T}^{\text {miss }}$ search strategy are so low that an effective field theory description might not be valid. The corresponding exclusion limits are therefore only indicative.

The $95 \%$ CL bounds that follow from our $j+E_{T}^{\text {miss }}$ search strategy are collected in table 4. As discussed at the end of section 2, mono-jet searches only allow to test the Wilson coefficient $c_{t}$ of the Yukawa-type DM-top operator in (2.1). It is evident from the shown results that the mono-jet bounds on $f / \sqrt{\left|c_{t}\right|}$ are not competitive with those 


\begin{tabular}{|c|ccccccc|}
\hline & \multicolumn{7}{|c|}{ DM mass } \\
Parameter & $70 \mathrm{GeV}$ & $100 \mathrm{GeV}$ & $200 \mathrm{GeV}$ & $300 \mathrm{GeV}$ & $400 \mathrm{GeV}$ & $500 \mathrm{GeV}$ & $1 \mathrm{TeV}$ \\
\hline$f / \sqrt{\left|c_{d}\right|}$ & $165 \mathrm{GeV}$ & $154 \mathrm{GeV}$ & $138 \mathrm{GeV}$ & $123 \mathrm{GeV}$ & $109 \mathrm{GeV}$ & $96 \mathrm{GeV}$ & $51 \mathrm{GeV}$ \\
$|\lambda|$ & 2.4 & 6.0 & $(23)$ & $(55)$ & $(107)$ & $(198)$ & $(2315)$ \\
$f / \sqrt{\left|c_{t}\right|}$ & $153 \mathrm{GeV}$ & $150 \mathrm{GeV}$ & $137 \mathrm{GeV}$ & $122 \mathrm{GeV}$ & $107 \mathrm{GeV}$ & $96 \mathrm{GeV}$ & $50 \mathrm{GeV}$ \\
$f / \sqrt{\mid d_{t_{R} \mid}}$ & $325 \mathrm{GeV}$ & $324 \mathrm{GeV}$ & $305 \mathrm{GeV}$ & $278 \mathrm{GeV}$ & $255 \mathrm{GeV}$ & $231 \mathrm{GeV}$ & $129 \mathrm{GeV}$ \\
\hline
\end{tabular}

Table 3. $95 \%$ CL bounds that derive from the $t X+E_{T}^{\text {miss }}$ search strategy described in section 4.1 for seven different DM masses. All bounds assume $3 \mathrm{ab}^{-1}$ of integrated luminosity collected at a $\mathrm{CM}$ energy of $14 \mathrm{TeV}$. Only the parameter shown in each line is taken into account, while all the remaining couplings in (2.1) are set to zero. See text for further explanations.

\begin{tabular}{|c|ccccccc|}
\hline & \multicolumn{7}{|c|}{ DM mass } \\
Parameter & $70 \mathrm{GeV}$ & $100 \mathrm{GeV}$ & $200 \mathrm{GeV}$ & $300 \mathrm{GeV}$ & $400 \mathrm{GeV}$ & $500 \mathrm{GeV}$ & $1 \mathrm{TeV}$ \\
\hline$f / \sqrt{\left|c_{t}\right|}$ & $96 \mathrm{GeV}$ & $95 \mathrm{GeV}$ & $90 \mathrm{GeV}$ & $81 \mathrm{GeV}$ & $74 \mathrm{GeV}$ & $65 \mathrm{GeV}$ & $36 \mathrm{GeV}$ \\
\hline
\end{tabular}

Table 4. As table 3 but for the $j+E_{T}^{\text {miss }}$ search strategy described in section 4.2 .

obtained from $t X+E_{T}^{\text {miss }}$. We add that neglecting the uncertainty on the shape of the $E_{T}^{\text {miss }}$ distribution (see section 4.2 ) in our $j+E_{T}^{\text {miss }}$ analysis would improve the given 95\% CL limits by around $35 \%$. However, even then the mono-jet limits on $f / \sqrt{\left|c_{t}\right|}$ fall short of the bounds obtained from our $t X+E_{T}^{\text {miss }}$ search strategy. Like in the case of the $t X+E_{T}^{\text {miss }}$ bounds, at high DM mass the $j+E_{T}^{\text {miss }}$ limits should only be taken as indicative, because an effective field theory description may not be applicable in this regime. Benchmark scenarios with more than one non-zero pNGB DM effective field theory coefficient $c_{i}, \lambda$ and $d_{j}$ are discussed in section 8 .

\section{Constraints from invisible Higgs decays at the LHC}

The terms in the first line of (2.1) will lead to invisible Higgs decays at tree level if this process is kinematically allowed, i.e. for $m_{\chi}<m_{h} / 2$. The relevant partial Higgs decay width reads

$$
\Gamma\left(h \rightarrow \chi^{*} \chi\right)=\frac{v^{2}}{16 \pi m_{h}} \sqrt{1-\frac{4 m_{\chi}^{2}}{m_{h}^{2}}}\left(\frac{m_{h}^{2} c_{d}}{f^{2}}-\lambda\right)^{2} .
$$

This formula can be used to translate experimental limits on the Higgs invisible branching ratio $\mathrm{BR}(h \rightarrow$ inv $)$ into constraints on $f / \sqrt{\left|c_{d}\right|}$ and $|\lambda|$. In fact, in the limit $m_{\chi} \ll m_{h} / 2$ one obtains the $95 \%$ CL exclusion limits

$$
\frac{f}{\sqrt{\left|c_{d}\right|}}>1.5 \mathrm{TeV}, \quad|\lambda|<7.2 \cdot 10^{-3} \quad(\text { LHC Run II })
$$

by employing the best existing LHC bound of BR $(h \rightarrow$ inv $)<0.11$ [89]. At the HL-LHC it may be possible to set a limit on the Higgs invisible branching ratio of $\mathrm{BR}(h \rightarrow$ inv $)<$ 


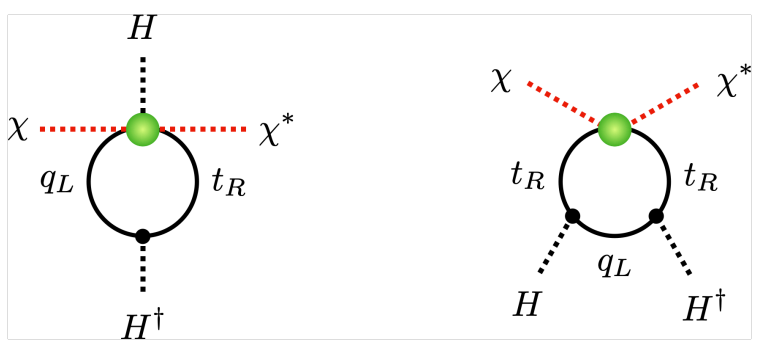

Figure 3. Left: an example of a diagram that describes the mixing of the Yukawa-type DM-top operator into the marginal Higgs portal operator. Right: example graph that could lead to a mixing of the current-current type DM-top operator into the DM-Higgs operators in (2.1). See text for further explanations.

$2.5 \cdot 10^{-2}[90]$. This implies that the bounds (6.2) may be improved to

$$
\frac{f}{\sqrt{\left|c_{d}\right|}}>2.2 \mathrm{TeV}, \quad|\lambda|<3.3 \cdot 10^{-3} \quad(\text { HL-LHC })
$$

Similar limits have also been given in [25]. Although the exclusion limits (6.2) and (6.3) have been derived under the assumption that either $c_{d}$ or $\lambda$ is non-zero but not both, the obtained stringent limits indicate that invisible Higgs decays are the main avenue to probe the pNGB DM couplings $c_{d}$ and $\lambda$ for DM masses $m_{\chi}<m_{h} / 2$.

At the loop level the first interaction term in the second line of (2.1) can also lead to invisible Higgs decays, because the Yukawa-type DM-top operator mixes into the marginal Higgs portal operator through fermion loops — see the left Feynman diagram in figure 3. Assuming that the marginal Higgs portal coupling vanishes at the scale $\mu_{f}=O(f)$, we obtain the following leading-logarithmic (LL) result

$$
\lambda=-\frac{3 m_{h}^{2} y_{t}^{2} c_{t}}{8 \pi^{2} f^{2}} \ln \frac{\mu_{f}}{\mu_{h}},
$$

for the marginal Higgs portal coupling at the EW scale $\mu_{h}=O\left(m_{h}\right)$. Notice that despite the fact that the contributions of the Yukawa-type DM-top operator to the invisible decays of the Higgs are loop suppressed the resulting constraints can still be important given the stringent bounds on BR $(h \rightarrow$ inv) that the HL-LHC is expected to set. For instance, taking as an example $c_{t}=1, y_{t} \simeq 0.94, \mu_{f}=f$ and $\mu_{h}=m_{h}$, we find numerically that the bound on $|\lambda|$ quoted in (6.3) leads to the limit

$$
f>450 \mathrm{GeV} \quad\left(c_{t}=1, \text { HL-LHC }\right)
$$

on the suppression scale of the Yukawa-type DM-top interactions introduced in (2.1). In contrast to the Yukawa-type DM-top operator, the current-current type DM-quark operators do not mix into the DM-Higgs operators appearing in (2.1) since the sum over all one-loop diagrams of the type shown on the right-hand side of figure 3 vanishes. The pNGB DM current-current type interactions therefore cannot be constrained by invisible Higgs decays even if $m_{\chi}<m_{h} / 2$. 


\section{Constraints from DM (in)direct detection and the relic density}

Even under the assumption that the interactions in (2.1) provide the leading new-physics effects at the scale $\mu_{f}$ at which the spin-0 fields emerge as composite pNGBs, the inclusion of radiative corrections can spoil this picture at the low energies probed in DM-nucleon scattering or DM annihilation (see [93-104] for further examples of relevant loop corrections in DM interactions). In fact, in the case at hand, we find that loop diagrams like those displayed in figure 4 induce couplings between $\mathrm{DM}$ and the $\mathrm{U}(1)_{Y}$ gauge boson or a pair of gluons. After EW symmetry breaking the DM gauge-boson interactions relevant for DM-nucleon scattering can be cast into the form

$$
\mathcal{L}_{\chi V}=\frac{i e c_{A}}{16 \pi^{2} f^{2}} \chi^{*} \stackrel{\leftrightarrow}{\partial_{\mu}} \chi \partial_{\nu} F^{\mu \nu}+\frac{g_{s}^{2} d_{G}}{16 \pi^{2} f^{2}}|\chi|^{2} G_{\mu \nu}^{a} G^{a, \mu \nu}
$$

where $e \simeq 0.3$ is the elementary electromagnetic charge, $g_{s} \simeq 1.2$ denotes the strong coupling constant and $F_{\mu \nu}$ represents the electromagnetic field strength tensor. The leading contributions to the Wilson coefficients of the operators in (7.1) read

$$
c_{A}=\frac{4}{3}\left(d_{q_{L}}+2 d_{t_{R}}-d_{b_{R}}\right) \ln \frac{\mu_{f}}{\mu_{h}}, \quad d_{G}=-\frac{c_{t}}{3} .
$$

Notice that the Wilson coefficient $c_{A}$ contains only the LL correction associated to operator mixing, while the result for $d_{G}$ corresponds to a finite matching correction obtained in the limit of infinite top-quark mass.

Including the tree-level contributions that arise from the marginal Higgs portal operator appearing in (2.1) as well as loop-induced interactions described by (7.2), the spinindependent (SI) DM-nucleon cross section can be written as

$$
\sigma_{\mathrm{SI}}=\frac{1}{\pi}\left(\frac{m_{\chi} m_{N}}{m_{\chi}+m_{N}}\right)^{2} \frac{1}{A^{2}}\left\{\frac{A m_{N}}{2 m_{\chi}}\left[\left(1-\frac{7 f_{T_{G}}^{N}}{9}\right) \frac{\lambda}{m_{h}^{2}}-\frac{2 f_{T_{G}}^{N} d_{G}}{9 f^{2}}\right]+\frac{Z e^{2} c_{A}}{16 \pi^{2} f^{2}}\right\}^{2} .
$$

Here $A(Z)$ is the mass (atomic) number of the nucleus, $m_{N} \simeq 0.939 \mathrm{GeV}$ denotes the average nucleon mass and $f_{T_{G}}^{N}=1-\sum_{q=u, d, s} f_{T_{q}}^{N} \simeq 0.89$ is the effective gluon-nucleon coupling, and its numerical value corresponds to the values $f_{T_{u}}^{N} \simeq 0.019, f_{T_{d}}^{N} \simeq 0.045$ and $f_{T_{s}}^{N} \simeq 0.043[105,106]$ for the quark-nucleon matrix elements. Furthermore, notice that the contribution in (7.3) proportional to $c_{A}$ arises from $t$-channel photon exchange and that the corresponding form factors simply count the number of valence quarks of the nucleons, i.e. $f_{V_{u}}^{p}=f_{V_{d}}^{n}=2$ and $f_{V_{d}}^{p}=f_{V_{u}}^{n}=1$.

For $m_{\chi}=100 \mathrm{GeV}$ the latest XENON1T 90\% CL upper limit on the SI DM-nucleon cross section reads $\sigma_{\mathrm{SI}}<9.12 \cdot 10^{-47} \mathrm{~cm}^{2}$ [107]. Using (7.3) with $A=131$ and $Z=54$ for xenon, this bound can be readily translated into limits on the Wilson coefficients of the relevant pNGB DM operators in (2.1). In the case of the marginal Higgs portal, we find in agreement with [25] the $90 \%$ CL exclusion limit

$$
|\lambda|<1.0 \cdot 10^{-2} .
$$



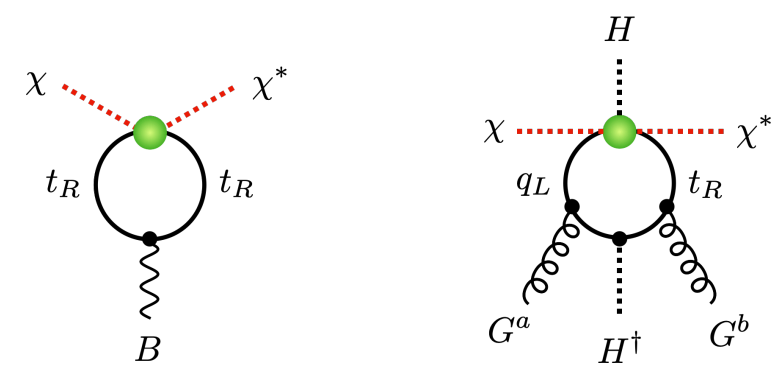

Figure 4. Left: example diagram that describes the LL contribution of the current-current type DM-fermion operators to the Wilson coefficient of the DM-photon operator appearing in (7.1). Right: a possible graph involving the insertion of the Yukawa-type DM-top operator that leads to a finite matching correction to the Wilson coefficient of the DM-gluon operator in (7.1). See text for further details.

Setting $c_{t}=1$ in (6.4) and (7.2) as well as using $\mu_{f}=f$ and $\mu_{h}=m_{h}$, and setting $d_{q_{L}}=d_{t_{R}}=d_{b_{R}}=1$ in (7.2), we obtain in addition the lower bounds

$$
\begin{array}{ll}
f>510 \mathrm{GeV} & \left(c_{t}=1\right), \\
f>1.3 \mathrm{TeV} & \left(d_{q_{L}}=d_{t_{R}}=d_{b_{R}}=1\right),
\end{array}
$$

on the suppression scale of the Yukawa-type and the current-current type DM-fermion interactions entering (2.1), respectively. Although we have considered in all cases only the effect of one type of pNGB DM operator at the scale $\mu_{f}$ at a time, the limits (7.4) and (7.5) show that the null results of the DM direct detection experiments generically allow to set stringent bounds on the Wilson coefficients of the marginal Higgs portal and the pNGB DM-fermion operators in (2.1). In contrast the derivative Higgs portal operator remains unconstrained by DM direct detection even after one-loop corrections are included in the calculation of the SI DM-nucleon cross section.

In order to understand the physics of DM indirect detection and thermal-freeze out in composite pNGB DM models, we first write the velocity-averaged cross section for annihilation of DM into a SM final state $X$ as

$$
\left\langle\sigma\left(\chi^{*} \chi \rightarrow X\right) v\right\rangle(T)=a_{X}+T b_{X} .
$$

Here $T$ denotes the DM temperature and thus the coefficient $a_{X}\left(b_{X}\right)$ describes the $s$-wave ( $p$-wave) contribution. Notice that in today's Universe $T_{0} \simeq 0$, while at freezeout $T_{f} \simeq m_{\chi} / 25$. This means that the $p$-wave coefficient $b_{X}$ can usually be neglected in the calculation of the DM indirect detection constraints, while it can be relevant in the computation of the relic abundance $\Omega_{\chi} h^{2}$, in particular if the corresponding $s$-wave coefficient $a_{X}$ is parametrically suppressed.

An example where such a parametric suppression is at work in the context of $(2.1)$ is the annihilation of DM into a bottom-antibottom quark pair, i.e. $\chi^{*} \chi \rightarrow b \bar{b}$. In this case, we find that the relevant $s$-wave and $p$-wave coefficients are well approximated by

$$
a_{b \bar{b}} \simeq \frac{3 m_{b}^{2}}{4 \pi}\left|\frac{1}{4 m_{\chi}^{2}-m_{h}^{2}+i m_{h} \Gamma_{h}}\left(\frac{4 m_{\chi}^{2} c_{d}}{f^{2}}-\lambda\right)\right|^{2}, \quad b_{b \bar{b}} \simeq \frac{3 m_{\chi}}{8 \pi} \frac{d_{q_{L}}^{2}+d_{b_{R}}^{2}}{f^{4}}
$$



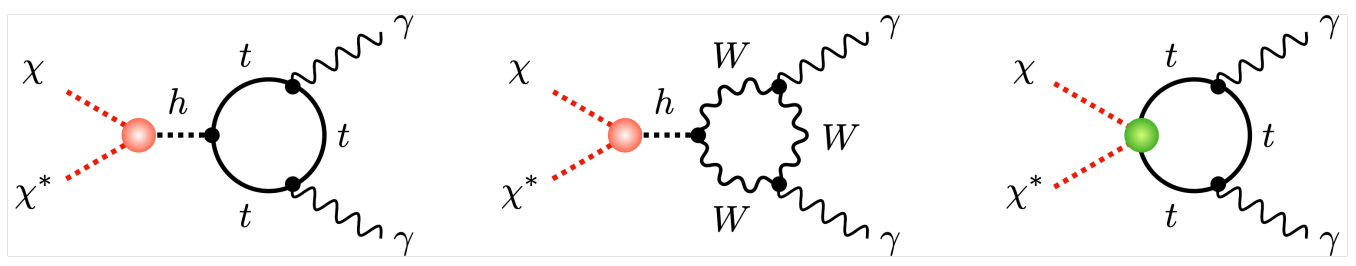

Figure 5. Example diagrams that lead to the process $\chi^{*} \chi \rightarrow \gamma \gamma$. Further details can be found in the text.

if the DM mass is sufficiently above the bottom-quark threshold at $m_{\chi}=m_{b} \simeq 4.2 \mathrm{GeV}$. In the above expression for $a_{b \bar{b}}$, the total decay width of the Higgs boson including contributions from $h \rightarrow \chi^{*} \chi$ (see section 6) is denoted by $\Gamma_{h}$. For $m_{b}<m_{\chi} \lesssim m_{W}$ with the $W$-boson mass $m_{W} \simeq 80.4 \mathrm{GeV}$, the $\chi^{*} \chi \rightarrow b \bar{b}$ channel generically provides the dominant mechanism to set $\Omega_{\chi} h^{2}$ in composite pNGB DM models described by (2.1). In fact, it turns out that for $m_{\chi} \ll m_{h} / 2$ the velocity suppression of the $p$-wave contribution in (7.7) is less severe than the bottom-mass suppression of the $s$-wave contribution in (7.7). The currentcurrent type DM-fermion operators introduced in (2.1) can therefore play an important role in thermal freeze-out for $m_{\chi}<m_{h} / 2$.

For $m_{\chi} \gtrsim m_{W}$ the $\chi^{*} \chi \rightarrow W^{+} W^{-}, Z Z, h h, t \bar{t}$ channels dominate DM annihilation. These processes all receive unsuppressed $s$-wave contributions, rendering the associated $p$-wave contributions phenomenologically irrelevant. For DM masses sufficiently far above the EW scale, we find the following approximations for the $s$-wave coefficients

$$
a_{X} \simeq \frac{N_{X} m_{\chi}^{2}}{4 \pi}\left[\frac{c_{d}}{f^{2}}-\frac{\lambda}{4 m_{\chi}^{2}}\right]^{2}, \quad a_{t \bar{t}} \simeq \frac{3 m_{t}^{2}}{4 \pi}\left[\frac{c_{d}+c_{t}}{f^{2}}-\frac{\lambda}{4 m_{\chi}^{2}}\right]^{2}
$$

where $X=W^{+} W^{-}, Z Z$, hh and $N_{W^{+} W^{-}}=2, N_{Z Z}=N_{h h}=1$. The above results can be shown to agree with the calculations performed in [108] after taking the limit of large DM mass. Notice that in this limit, DM annihilation to $W$ and $Z$ bosons reduces to three times the contribution from annihilation to the Higgs boson, as expected in the $\mathrm{SU}(2)_{L} \times \mathrm{U}(1)_{Y}$ symmetric limit. Given that the size of the marginal Higgs portal coupling $\lambda$ is strongly constrained by DM direct detection (see (7.4)), the expressions (7.8) also imply that in viable composite pNGB DM models the derivative Higgs portal operator generically provides the dominant contribution to DM annihilation for $m_{\chi} \gg m_{t}$. As a result thermal freeze-out becomes a model-independent prediction in this limit, in the sense that the value of $\Omega_{\chi} h^{2}$ to first approximation only depends on $m_{\chi}$ and $f / \sqrt{\left|c_{d}\right|}$.

In addition to the DM annihilation channels discussed so far, DM annihilation into mono-chromatic photons can provide a relevant indirect-detection signature in composite pNGB DM models. As shown in figure 5, this signature receives two types of contributions. The first is associated to $s$-channel exchange of a Higgs boson with subsequent decay of the Higgs into a pair of photons, i.e. $\chi^{*} \chi \rightarrow h \rightarrow \gamma \gamma$, and proceeds through the insertion of a DM-Higgs operator and a loop of top quarks (left diagram) or $W$ bosons (middle diagram). 
The corresponding form factors describing fermion and gauge-boson loops are given by

$$
\begin{aligned}
& F_{\psi}(\tau)=\frac{3 \tau}{2}\left[1+(1-\tau) \arctan ^{2} \frac{1}{\sqrt{\tau-1}}\right], \\
& F_{V}(\tau)=\frac{1}{7}\left[2+3 \tau+3 \tau(2-\tau) \arctan ^{2} \frac{1}{\sqrt{\tau-1}}\right],
\end{aligned}
$$

respectively, and are normalised such that $F_{\psi}(\infty)=F_{V}(\infty)=1$. The second type of contributions involves the insertion of the Yukawa-type DM-top operator introduced in (2.1) and leads directly to the $\chi^{*} \chi \rightarrow \gamma \gamma$ transition via a top-quark loop (right diagram in figure 5). Including both types of contributions, the $s$-wave coefficient corresponding to $\chi^{*} \chi \rightarrow \gamma \gamma$ annihilation can be written as

$a_{\gamma \gamma}=\frac{\alpha^{2} m_{\chi}^{2}}{8 \pi^{3}}\left|\frac{1}{4 m_{\chi}^{2}-m_{h}^{2}+i m_{h} \Gamma_{h}}\left(\frac{4 m_{\chi}^{2} c_{d}}{f^{2}}-\lambda\right)\left[\frac{8 F_{\psi}\left(\tau_{t}\right)}{9}-\frac{7 F_{V}\left(\tau_{W}\right)}{2}\right]+\frac{8 c_{t}}{9 f^{2}} F_{\psi}\left(\tau_{t}\right)\right|^{2}$,

where $\tau_{i}=m_{i}^{2} / m_{\chi}^{2}-i \varepsilon$ with $\varepsilon$ being a positive infinitesimal real number. Notice that the $s$-channel Higgs exchange contribution in (7.10) is resonantly enhanced at $m_{\chi}=m_{h} / 2$, and as a result the DM indirect detection constraints from the observation of $\gamma$-ray lines are generically most stringent in the vicinity of the Higgs pole.

Based on (7.6) to (7.8), the present abundance of DM in the Universe is approximately given by the following formula

$$
\frac{\Omega_{\chi} h^{2}}{0.12} \simeq \frac{3 \cdot 10^{-26} \mathrm{~cm}^{3} / \mathrm{s}}{\langle\sigma v\rangle_{f}}, \quad\langle\sigma v\rangle_{f}=\frac{1}{2} \sum_{X}\left\langle\sigma\left(\chi^{*} \chi \rightarrow X\right) v\right\rangle\left(T_{f}\right),
$$

where the sum over $X$ involves all annihilation channels that are kinematically accessible at a given DM mass. Notice that the factor of $1 / 2$ in the definition of $\langle\sigma v\rangle_{f}$ takes into account that DM is not self-conjugate in our case. The same factor of $1 / 2$ appears when one calculates the $\gamma$-ray flux from the annihilation cross section (7.10). While (7.11) represents a useful expression to estimate $\Omega_{\chi} h^{2}$, we will use micrOMEGAs [109] in our numerical analysis of the constraints on the pNGB DM parameter space following from the requirement to reproduce the relic abundance of $\Omega_{\chi} h^{2}=0.120 \pm 0.001$ as measured by PLANCK [110]. micrOMEGAs is also used to determine the DM indirect detection exclusion limits.

\section{Discussion}

In figures 6 to 8 we summarise the most important constraints in the $m_{\chi}-f$ plane for the three benchmark models with $c_{d}=1, c_{d}=c_{t}=1$ and $c_{d}=d_{q_{L}}=d_{t_{R}}=d_{b_{R}}=1$. Similar benchmark models have also been considered in [25]. The pNGB DM effective field theory parameters not shown in the headline of each figure are set to zero to obtain the displayed results. The dark red and blue regions are excluded by the projected HL-LHC limit on the Higgs invisible branching ratio of $\mathrm{BR}(h \rightarrow \mathrm{inv})<2.5 \cdot 10^{-2}[90]$ and by the 90\% CL bounds on the SI DM-nucleon cross section set by XENON1T [107], respectively. The vertical grey bands indicate the DM mass ranges that are excluded at 95\% CL by 


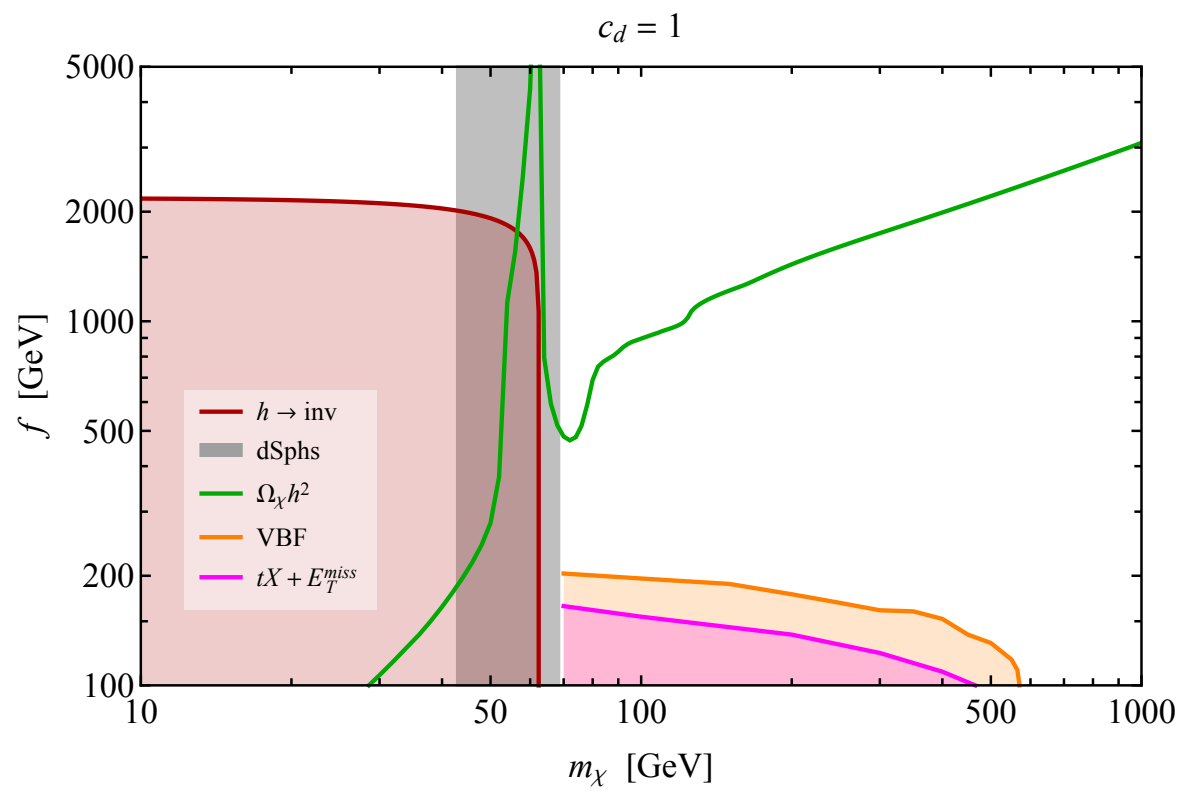

Figure 6. Constraints in the $m_{\chi}-f$ plane for the derivative Higgs portal model. The pNGB DM effective field theory parameters not shown in the headline of the plot are set to zero to obtain the displayed results. The dark red region is excluded by the projected HL-LHC 95\% CL limit on the Higgs invisible branching ratio of $\mathrm{BR}(h \rightarrow$ inv $)<2.5 \cdot 10^{-2}[90]$. The vertical grey band displays the DM mass range that is excluded at $95 \%$ CL by the dSphs analysis of Fermi-LAT and DES [111] assuming $\chi^{*} \chi \rightarrow b \bar{b}$ annihilation. The green curve corresponds to the value $\Omega_{\chi} h^{2}=0.12$ of the DM relic density as determined by PLANCK [110]. In the parameter space above the green curves the Universe is overclosed. The orange region indicates the 95\% CL exclusion limit derived in [25] from a study of off-shell invisible Higgs production in the VBF channel at the HL-LHC, while the magenta region represents the corresponding exclusion limit obtained by our $t X+E_{T}^{\text {miss }}$ search strategy. Consult the main text for further details.

the $\gamma$-ray observations of dwarf spheroidal galaxies (dSphs) of the Fermi-LAT and DES collaborations in [111]. The used experimental bounds assume DM annihilation into $b \bar{b}$ final states and that the measured relic density is reproduced. The constraints that follow from the latest Fermi-LAT search for $\gamma$-ray lines [112] lead to weaker constraints on the DM mass of $62.5 \mathrm{GeV} \lesssim m_{\chi} \lesssim 64 \mathrm{GeV}$ compared to $\chi^{*} \chi \rightarrow b \bar{b}$ even if a favourable DM distribution (such as an adiabatically contracted Navarro-Frenk-White profile [113]) is used to calculate the limits. These bounds are hence not shown in figures 6 to 9 . The green curves correspond to the PLANCK value $\Omega_{\chi} h^{2}=0.12$ [110] of the DM relic abundance. The orange regions displayed in the figures correspond to the $95 \%$ CL exclusion limits found in [25] from a HL-LHC study of off-shell invisible Higgs production in the VBF channel. The magenta domains finally correspond to the $95 \%$ CL constraints obtained by the $t X+E_{T}^{\text {miss }}$ analysis strategy discussed in section 4.1.

In the case of the derivative Higgs portal model, one observes from figure 6 that in the Higgs on-shell region corresponding to $m_{\chi}<m_{h} / 2$, HL-LHC measurements of invisible Higgs decays exclude large parts of the parameter space that leads to the correct DM relic density via standard thermal freeze-out. Only a narrow corridor around the Higgs resonance 


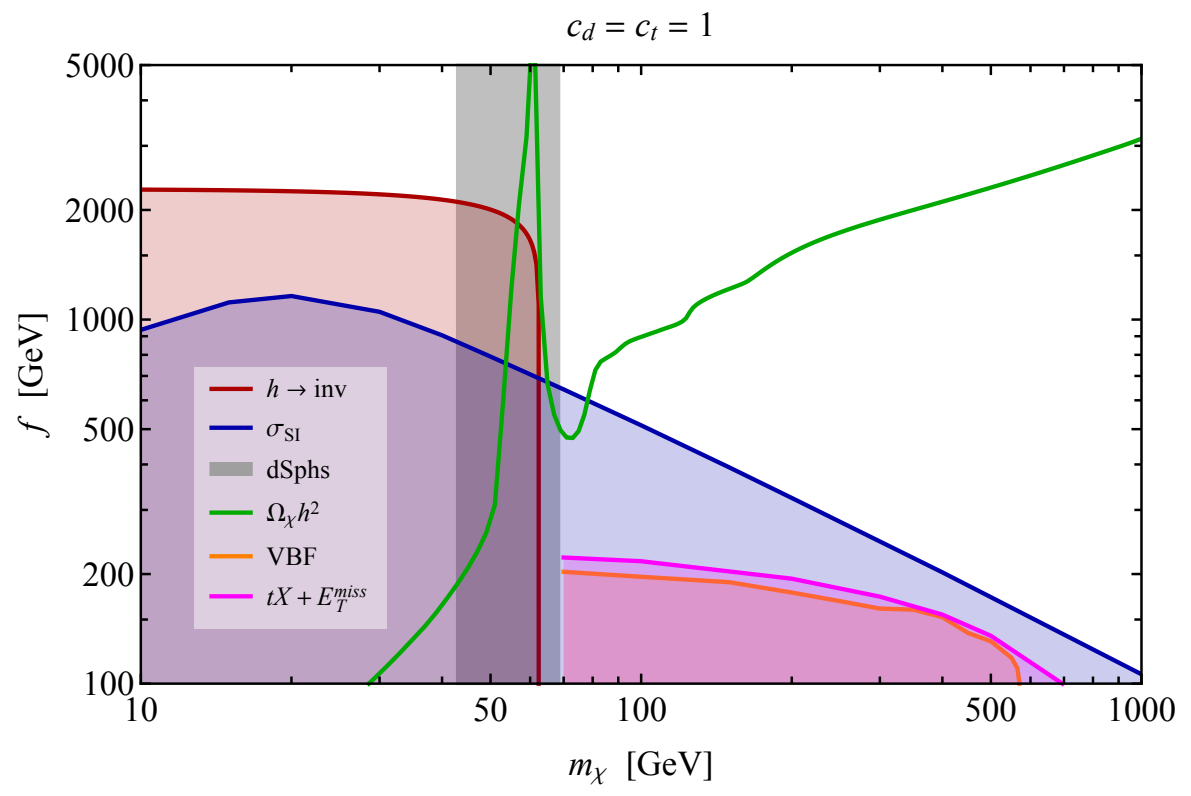

Figure 7. As figure 6 but for the pNGB DM benchmark model with $c_{d}=c_{t}=1$. The blue region is excluded by the $90 \%$ CL bound on the SI DM-nucleon cross section $\sigma_{\mathrm{SI}}$ as determined by XENON1T [107].

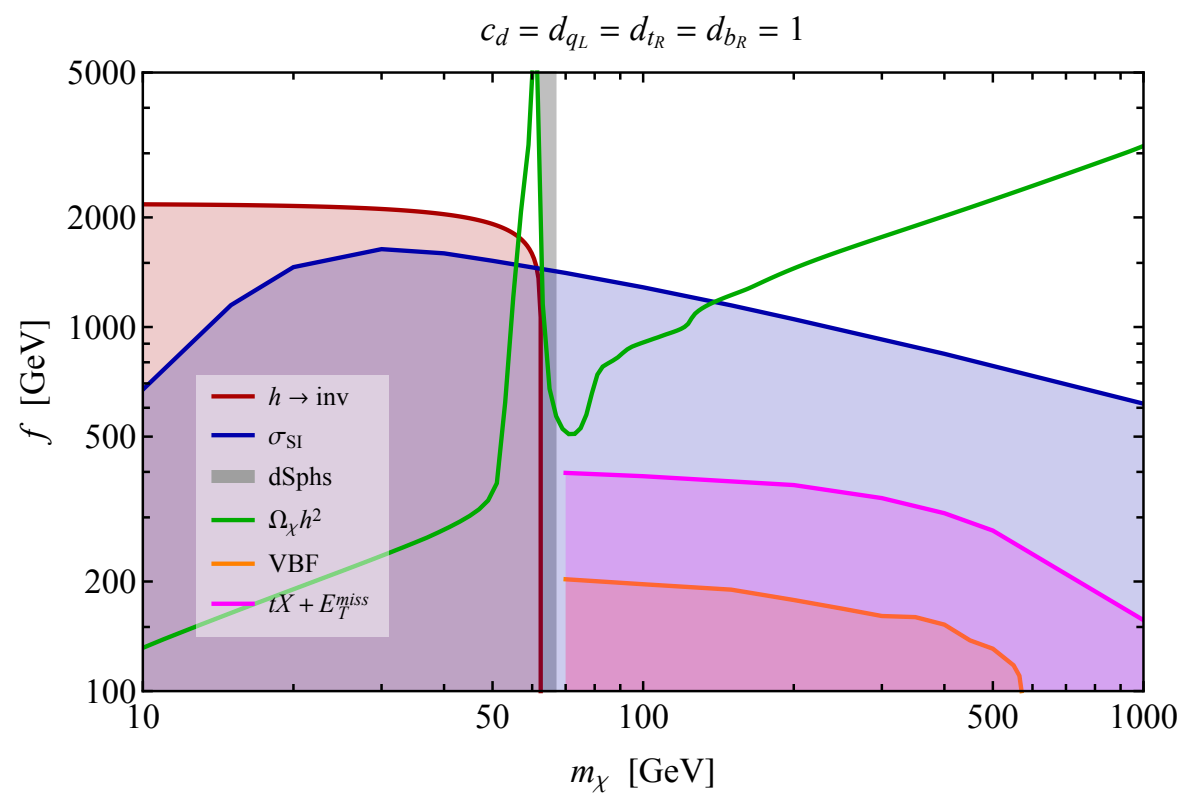

Figure 8. As figure 7 but for the pNGB DM benchmark model with $c_{d}=d_{q_{L}}=d_{t_{R}}=d_{b_{R}}=1$. 


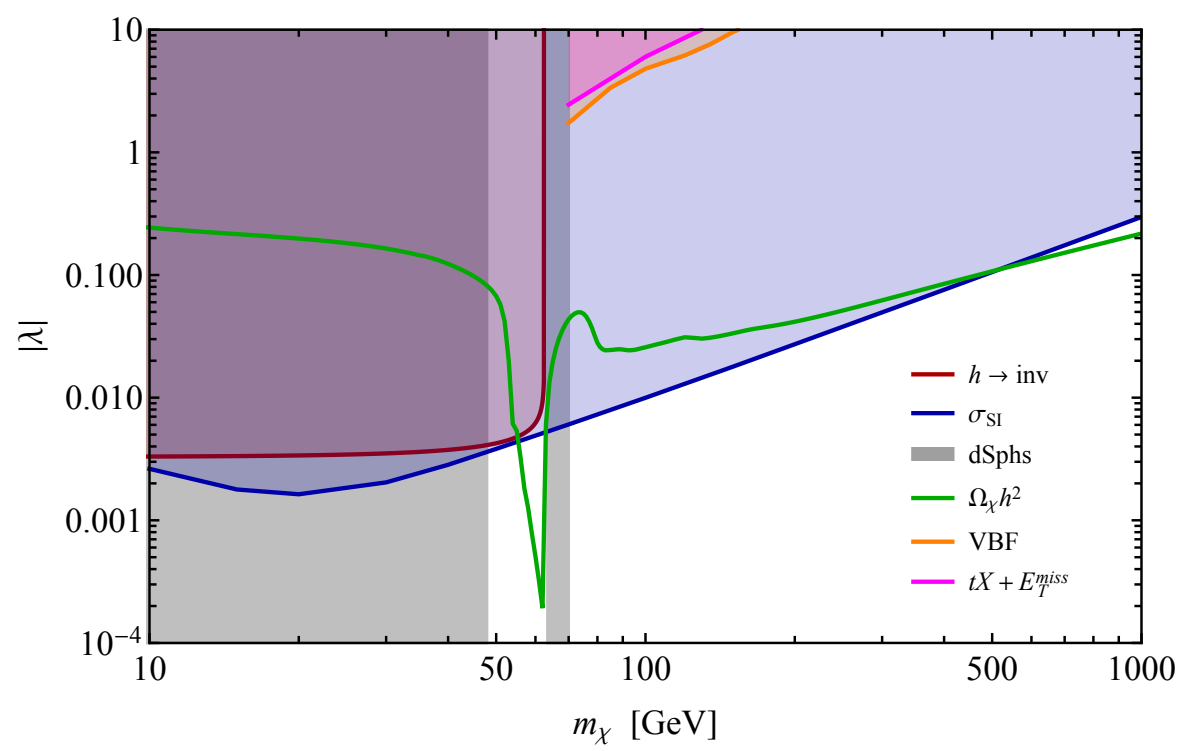

Figure 9. Constraints in the $m_{\chi}-|\lambda|$ plane for the marginal Higgs portal model. Apart from the fact that in the parameter space below the green curve the Universe is overclosed, the meaning and colour coding of the shown constraints resemble those of figure 7 .

survives this constraint, which is however excluded by DM indirect detection measurements. Since the DM-nucleon scattering rate is momentum suppressed, the stringent limits from DM direct detection experiments do not put constraints on the pNGB DM benchmark model with only $c_{d}=1$. This opens up the possibility to test such models with $m_{\chi}>$ $m_{h} / 2$ using mono- $X$ searches at the HL-LHC, however only if these models lead to a DM underabundance, i.e. $\Omega_{\chi} h^{2}<0.12$. Given that the VBF limits taken from [25] are around $30 \%$ better than the $t X+E_{T}^{\text {miss }}$ bounds on $f$, the best test of the derivative Higgs portal model in the Higgs off-shell region seems to be provided by invisible Higgs production in the VBF channel. In this context it is however important to realise that the study [25] assumes a systematic uncertainty on the relevant SM background of $1 \%$, while the shown $t X+E_{T}^{\text {miss }}$ exclusion is based on a systematic uncertainty on the relevant SM background of $15 \%$ (see section 4.1). Assuming a reduction of the systematic background uncertainties in $t X+E_{T}^{\text {miss }}$ down to $5 \%$ would bring the VBF and $t X+E_{T}^{\text {miss }}$ exclusion limits closer together. See appendix A for details.

As can be seen from figures 7 and 8 , the HL-LHC potential to test viable models through mono- $X$ searches is less favourable in the case of the pNGB DM benchmarks with $c_{d}=c_{t}=1$ or $c_{d}=d_{q_{L}}=d_{t_{R}}=d_{b_{R}}=1$ since in these cases the limits from DM direct detection, though loop suppressed, turn out to be still severe. In the first case the LL corrections to $\lambda$ in (6.4) and the finite matching correction to $d_{G}$ in (7.2) are both relevant, while in the second case the LL corrections to $c_{A}$ in (7.2) play an essential role in determining the correct DM direct detection limits. The above LL corrections have not been discussed in the work [25], but it is known (see for example [96-98, 100-104]) that the inclusion of radiative corrections can have important effects in the calculation of $\sigma_{\mathrm{SI}}$. 
Comparing the VBF and $t X+E_{T}^{\text {miss }}$ constraints, one sees that in both cases $c_{d}=c_{t}=1$ and $c_{d}=d_{q_{L}}=d_{t_{R}}=d_{b_{R}}=1$ the limits on $f$ derived here are stronger than the bounds that have been obtained in [25]. This result follows straightforwardly from the fact that invisible VBF Higgs off-shell production is only sensitive to $c_{d}$, while the $t X+E_{T}^{\text {miss }}$ signature receives contributions from $c_{d}$ but also from $c_{t}, d_{q_{L}}$ and $d_{t_{R}}$.

In figure 9 we finally summarise the constraints on the marginal Higgs portal model set by DM (in)direct detection experiments, the relic density and future HL-LHC searches. One observes that the constraints on $|\lambda|$ from DM direct detection and the HL-LHC are comparable for DM masses $m_{\chi}<m_{h} / 2$. However, in the case $m_{\chi}>m_{h} / 2$ the bounds that follow from $\sigma_{\mathrm{SI}}$ are by more than two orders of magnitude stronger than those that one can hope to obtain at the HL-LHC from mono- $X$ searches. Like in the case of the derivative Higgs portal model, off-shell invisible Higgs production in the VBF channel [25] again seems to be the best way to probe the marginal Higgs portal model at the LHC if $m_{\chi}>$ $m_{h} / 2$. This conclusion once more depends on the actual size of systematic background uncertainties of the VBF and $t X+E_{T}^{\text {miss }}$ channels in the HL-LHC environment. Combining the two mono- $X$ channels as done in the case of the LHC searches for the invisible Higgs boson decays (see for instance [89, 114-116]) can be expected to improve the ultimate HL-LHC reach. Performing an actual combination of the VBF and $t X+E_{T}^{\text {miss }}$ channels is however beyond the scope of this article. We add that the potential of the high-energy option of the LHC, the future circular hadron-hadron collider, the compact linear collider and a muon collider in constraining the marginal Higgs portal through VBF off-shell Higgs production has been studied in the article [25]. See also [117-121] for similar analyses.

pNGB DM models in which both the SM Higgs boson as well as the DM candidate are composites of a $\mathrm{TeV}$-scale strongly-coupled sector provide a simultaneous explanation of the EW hierarchy problem and the DM puzzle. Key features in this class of beyond the SM theories are that the SM Higgs boson and the DM particle are both naturally light, and that the leading coupling between DM and the SM is the derivative Higgs portal. This portal is strongly suppressed in the regime of small momentum transfer that is probed by DM scattering with heavy nuclei, making this type of WIMP easily compatible with the existing strong constraints from DM direct detection experiments. At the same time, the interaction strength of DM annihilation turns out to be in the right range to obtain the observed relic density through thermal freeze-out without tuning. However, as we have shown in our work, this simple and attractive picture can be significantly altered by explicit symmetry breaking effects that lead to pNGB DM interactions beyond the derivative Higgs portal. In fact, once radiative effects are taken into account, only pNGB DM realisations of the form (2.1) with $c_{d} \neq 0$ and all other pNGB DM effective field theory parameters sufficiently small typically survive the constraints from DM direct detection experiments. In such scenarios, collider searches for DM production are the only known direct way to explore the pNGB DM parameter space. If the DM candidate is kinematically accessible, searches for invisible Higgs boson decays play a key role in such explorations, while DM masses above the Higgs threshold can be probed by studying mono- $X$ signatures. In our article, we have extended the earlier study of off-shell invisible Higgs production via VBF [25] by developing a search strategy that allows to probe pNGB DM using $t X+E_{T}^{\text {miss }}$ signatures. 
The $t X+E_{T}^{\text {miss }}$ channels are complementary to VBF Higgs production since they are able to test pNGB DM interactions like the Yukawa-type DM-top coupling and the currentcurrent type interactions in (2.1) that are not accessible via the latter mode. Together with [25] the work presented here provides the blueprints to search for pNGB DM at the LHC, and we encourage ATLAS and CMS to perform dedicated experimental searches and interpretations of the relevant mono- $X$ signatures.

\section{Acknowledgments}

We thank Maximilian Ruhdorfer, Ennio Salvioni and Andreas Weiler for useful discussions, for their helpful comments on this manuscript and for providing us with the computer codes employed in their paper [25] to determine the DM indirect detection and relic density constraints on composite pNGB DM models. Our analytic calculations made use of the Mathematica packages FeynArts [122], FormCalc [123, 124] and FeynCalc [125-127]. This research has been partially supported by the Collaborative Research Center SFB1258.

\section{A Supplementary material}

In this appendix we present HL-LHC projections based on alternative more aggressive assumptions about the systematic uncertainties of our $t X+E_{T}^{\text {miss }}$ search strategy. Anticipating improvements in detector performance and modelling of SM background processes, we assume that the systematic uncertainties on the number of expected events in the signal regions SR1, SR2 and SR3 are reduced from $15 \%$ to $5 \%$ and $1 \%$. In figure 10 we show the 95\% CL constraints in the $m_{\chi}-f$ plane for the derivative Higgs portal model (upper panel) and in the $m_{\chi^{-}}-\lambda \mid$ plane for the marginal Higgs portal model (lower panel). The orange regions indicate the exclusion limits derived in the study of off-shell invisible Higgs production in the VBF channel [25]. The displayed results assume a $1 \%$ systematic uncertainty on the relevant SM backgrounds. For comparison we show in magenta the 95\% CL limits that derive from the $t X+E_{T}^{\text {miss }}$ search strategy discussed in section 4.1. Here the solid, dashed and dotted contours correspond to assumed systematic background uncertainties of $15 \%, 5 \%$ and $1 \%$, respectively. It is evident from both panels that reducing the systematic uncertainties from $15 \%$ to $5 \%$ has a visible impact on the obtained $t X+E_{T}^{\text {miss }}$ exclusion limits, while a further uncertainty reduction to $1 \%$ has only a minor effect on the bounds in the shown parameter planes. Notice that a reduction of the systematic uncertainties to $5 \%$ may be possible given the steady progress of both experiment and theory. In the case of the marginal Higgs portal, such an improvement would lead to a reach in the $t X+E_{T}^{\text {miss }}$ channel that is very similar to the one of VBF invisible Higgs production in the off-shell region. 

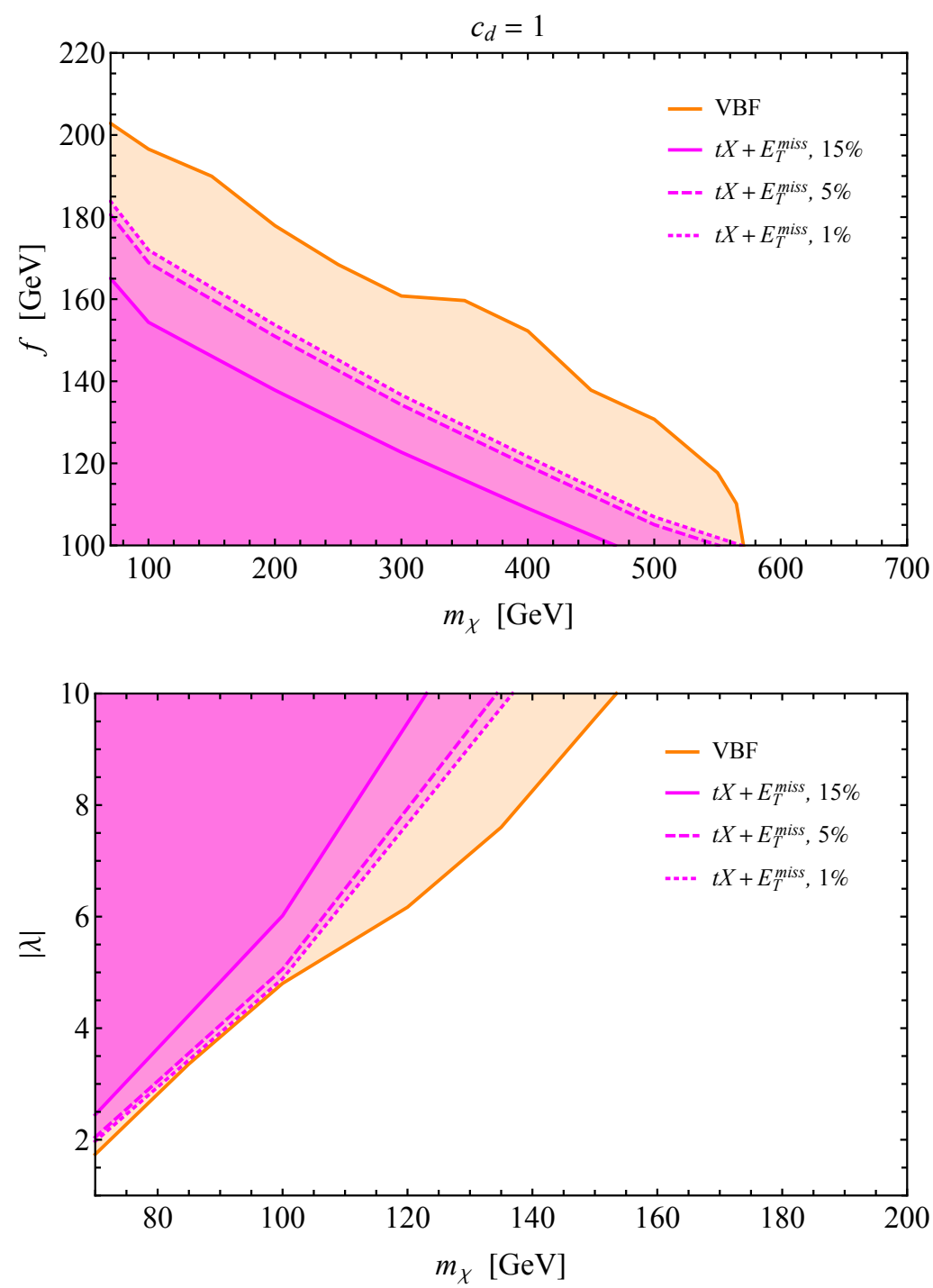

Figure 10. 95\% CL constraints in the $m_{\chi}-f$ plane for the derivative Higgs portal model (upper panel) and in the $m_{\chi}-|\lambda|$ plane for the marginal Higgs portal model (lower panel). The orange regions correspond to the 95\% CL exclusion limits determined in [25] from a HL-LHC study of off-shell invisible Higgs production in the VBF channel, while the magenta contours represent the results of our $t X+E_{T}^{\text {miss }}$ search assuming a systematic background uncertainty of $15 \%$ (solid curves), $5 \%$ (dashed curves) and $1 \%$ (dotted curves). 
Open Access. This article is distributed under the terms of the Creative Commons Attribution License (CC-BY 4.0), which permits any use, distribution and reproduction in any medium, provided the original author(s) and source are credited.

\section{References}

[1] M. Klasen, M. Pohl and G. Sigl, Indirect and direct search for dark matter, Prog. Part. Nucl. Phys. 85 (2015) 1 [arXiv:1507.03800] [InSPIRE].

[2] M. Schumann, Direct Detection of WIMP Dark Matter: Concepts and Status, J. Phys. G 46 (2019) 103003 [arXiv:1903.03026] [INSPIRE].

[3] ATLAS collaboration, Constraints on mediator-based dark matter and scalar dark energy models using $\sqrt{s}=13 \mathrm{TeV}$ pp collision data collected by the ATLAS detector, JHEP 05 (2019) 142 [arXiv: 1903.01400] [INSPIRE].

[4] M. Frigerio, A. Pomarol, F. Riva and A. Urbano, Composite Scalar Dark Matter, JHEP 07 (2012) 015 [arXiv: 1204.2808] [INSPIRE].

[5] V. Barger, P. Langacker, M. McCaskey, M. Ramsey-Musolf and G. Shaughnessy, Complex Singlet Extension of the Standard Model, Phys. Rev. D 79 (2009) 015018 [arXiv: 0811.0393] [INSPIRE].

[6] V. Barger, M. McCaskey and G. Shaughnessy, Complex Scalar Dark Matter vis-à-vis CoGeNT, DAMA/LIBRA and XENON100, Phys. Rev. D 82 (2010) 035019 [arXiv: 1005.3328] [INSPIRE].

[7] M. Chala, $h \rightarrow \gamma \gamma$ excess and Dark Matter from Composite Higgs Models, JHEP 01 (2013) 122 [arXiv:1210.6208] [INSPIRE].

[8] D. Marzocca and A. Urbano, Composite Dark Matter and LHC Interplay, JHEP 07 (2014) 107 [arXiv: 1404.7419] [INSPIRE].

[9] J. Barnard, T. Gherghetta, T.S. Ray and A. Spray, The Unnatural Composite Higgs, JHEP 01 (2015) 067 [arXiv: 1409.7391] [InSPIRE].

[10] N. Fonseca, R. Zukanovich Funchal, A. Lessa and L. Lopez-Honorez, Dark Matter Constraints on Composite Higgs Models, JHEP 06 (2015) 154 [arXiv:1501.05957] [INSPIRE].

[11] I. Brivio et al., Non-linear Higgs portal to Dark Matter, JHEP 04 (2016) 141 [arXiv: 1511.01099] [INSPIRE].

[12] M. Kim, S.J. Lee and A. Parolini, WIMP Dark Matter in Composite Higgs Models and the Dilaton Portal, arXiv: 1602.05590 [INSPIRE].

[13] M. Chala, G. Nardini and I. Sobolev, Unified explanation for dark matter and electroweak baryogenesis with direct detection and gravitational wave signatures, Phys. Rev. D 94 (2016) 055006 [arXiv: 1605.08663] [INSPIRE].

[14] D. Barducci et al., Monojet searches for momentum-dependent dark matter interactions, JHEP 01 (2017) 078 [arXiv: 1609.07490] [INSPIRE].

[15] Y. Wu, T. Ma, B. Zhang and G. Cacciapaglia, Composite Dark Matter and Higgs, JHEP 11 (2017) 058 [arXiv: 1703.06903] [INSPIRE].

[16] R. Balkin, M. Ruhdorfer, E. Salvioni and A. Weiler, Charged Composite Scalar Dark Matter, JHEP 11 (2017) 094 [arXiv:1707.07685] [INSPIRE]. 
[17] R. Balkin, G. Perez and A. Weiler, Little composite dark matter, Eur. Phys. J. C 78 (2018) 104 [arXiv: 1707.09980] [INSPIRE].

[18] C. Gross, O. Lebedev and T. Toma, Cancellation Mechanism for Dark-Matter-Nucleon Interaction, Phys. Rev. Lett. 119 (2017) 191801 [arXiv:1708.02253] [INSPIRE].

[19] T. Alanne, D. Buarque Franzosi, M.T. Frandsen and M. Rosenlyst, Dark matter in (partially) composite Higgs models, JHEP 12 (2018) 088 [arXiv:1808.07515] [INSPIRE].

[20] R. Balkin, M. Ruhdorfer, E. Salvioni and A. Weiler, Dark matter shifts away from direct detection, JCAP 11 (2018) 050 [arXiv:1809.09106] [INSPIRE].

[21] K. Ishiwata and T. Toma, Probing pseudo Nambu-Goldstone boson dark matter at loop level, JHEP 12 (2018) 089 [arXiv:1810.08139] [INSPIRE].

[22] K. Huitu, N. Koivunen, O. Lebedev, S. Mondal and T. Toma, Probing pseudo-Goldstone dark matter at the LHC, Phys. Rev. D 100 (2019) 015009 [arXiv: 1812.05952] [INSPIRE].

[23] D. Karamitros, Pseudo Nambu-Goldstone Dark Matter: Examples of Vanishing Direct Detection Cross Section, Phys. Rev. D 99 (2019) 095036 [arXiv:1901.09751] [INSPIRE].

[24] A. Davoli, A. De Simone, D. Marzocca and A. Morandini, Composite 2HDM with singlets: a viable dark matter scenario, JHEP 10 (2019) 196 [arXiv:1905.13244] [INSPIRE].

[25] M. Ruhdorfer, E. Salvioni and A. Weiler, A Global View of the Off-Shell Higgs Portal, SciPost Phys. 8 (2020) 027 [arXiv: 1910.04170] [InSPIRE].

[26] M. Ramos, Composite dark matter phenomenology in the presence of lighter degrees of freedom, JHEP 07 (2020) 128 [arXiv:1912.11061] [INSPIRE].

[27] C. Arina, A. Beniwal, C. Degrande, J. Heisig and A. Scaffidi, Global fit of pseudo-Nambu-Goldstone Dark Matter, JHEP 04 (2020) 015 [arXiv: 1912.04008] [INSPIRE].

[28] Y. Abe, T. Toma and K. Tsumura, Pseudo-Nambu-Goldstone dark matter from gauged $\mathrm{U}(1)_{B-L}$ symmetry, JHEP 05 (2020) 057 [arXiv: 2001. 03954] [INSPIRE].

[29] N. Okada, D. Raut and Q. Shafi, Pseudo-Goldstone dark matter in a gauged B - L extended standard model, Phys. Rev. D 103 (2021) 055024 [arXiv:2001.05910] [InSPIRE].

[30] C.-Y. Xing, L.-X. Xu and S.-h. Zhu, Softly shifting away from dark matter direct detection, Phys. Rev. D 103 (2021) 113006 [arXiv:2011.06264] [INSPIRE].

[31] N. Okada, D. Raut, Q. Shafi and A. Thapa, Pseudo-Goldstone Dark Matter in SO(10), arXiv:2105.03419 [INSPIRE].

[32] L. Coito, C. Faubel, J. Herrero-Garcia and A. Santamaria, Dark matter from a complex scalar singlet: The role of dark CP and other discrete symmetries, arXiv:2106.05289 [INSPIRE].

[33] U. Haisch, M. Ruhdorfer, E. Salvioni, E. Venturini and A. Weiler, Singlet night in Feynman-ville: one-loop matching of a real scalar, JHEP 04 (2020) 164 [Erratum ibid. 07 (2020) 066] [arXiv: 2003.05936] [INSPIRE].

[34] U. Haisch, M. Ruhdorfer, E. Salvioni, E. Venturini and A. Weiler, in preparation.

[35] CMS collaboration, Search for dark matter produced in association with heavy-flavor quark pairs in proton-proton collisions at $\sqrt{s}=13 \mathrm{TeV}$, Eur. Phys. J. C 77 (2017) 845 [arXiv: 1706. 02581] [INSPIRE]. 
[36] ATLAS collaboration, Search for dark matter produced in association with bottom or top quarks in $\sqrt{s}=13 \mathrm{TeV}$ pp collisions with the ATLAS detector, Eur. Phys. J. C $\mathbf{7 8}$ (2018) 18 [arXiv:1710.11412] [INSPIRE].

[37] ATLAS collaboration, Search for new phenomena in final states with b-jets and missing transverse momentum in $\sqrt{s}=13 \mathrm{TeV}$ pp collisions with the ATLAS detector, JHEP 05 (2021) 093 [arXiv:2101.12527] [INSPIRE].

[38] T. Lin, E.W. Kolb and L.-T. Wang, Probing dark matter couplings to top and bottom quarks at the LHC, Phys. Rev. D 88 (2013) 063510 [arXiv:1303.6638] [INSPIRE].

[39] M.R. Buckley, D. Feld and D. Goncalves, Scalar Simplified Models for Dark Matter, Phys. Rev. D 91 (2015) 015017 [arXiv: 1410.6497] [INSPIRE].

[40] U. Haisch and E. Re, Simplified dark matter top-quark interactions at the LHC, JHEP 06 (2015) 078 [arXiv: 1503.00691] [INSPIRE].

[41] C. Arina et al., A comprehensive approach to dark matter studies: exploration of simplified top-philic models, JHEP 11 (2016) 111 [arXiv:1605.09242] [INSPIRE].

[42] U. Haisch, P. Pani and G. Polesello, Determining the CP nature of spin-0 mediators in associated production of dark matter and $t \bar{t}$ pairs, JHEP 02 (2017) 131 [arXiv:1611.09841] [INSPIRE].

[43] CMS collaboration, Search for top squarks and dark matter particles in opposite-charge dilepton final states at $\sqrt{s}=13 \mathrm{TeV}$, Phys. Rev. D 97 (2018) 032009 [arXiv:1711.00752] [INSPIRE].

[44] CMS collaboration, Search for dark matter particles produced in association with a top quark pair at $\sqrt{s}=13 \mathrm{TeV}$, Phys. Rev. Lett. 122 (2019) 011803 [arXiv:1807.06522] [INSPIRE].

[45] U. Haisch and G. Polesello, Searching for production of dark matter in association with top quarks at the LHC, JHEP 02 (2019) 029 [arXiv:1812.00694] [INSPIRE].

[46] CMS collaboration, Search for dark matter produced in association with a single top quark or a top quark pair in proton-proton collisions at $\sqrt{s}=13 \mathrm{TeV}$, JHEP 03 (2019) 141 [arXiv: 1901.01553] [INSPIRE].

[47] ATLAS collaboration, Search for new phenomena with top quark pairs in final states with one lepton, jets, and missing transverse momentum in pp collisions at $\sqrt{s}=13 \mathrm{TeV}$ with the ATLAS detector, JHEP 04 (2021) 174 [arXiv:2012.03799] [INSPIRE].

[48] ATLAS collaboration, Search for new phenomena in events with two opposite-charge leptons, jets and missing transverse momentum in pp collisions at $\sqrt{\mathrm{s}}=13 \mathrm{TeV}$ with the ATLAS detector, JHEP 04 (2021) 165 [arXiv:2102.01444] [INSPIRE].

[49] G. Durieux, J. Gu, E. Vryonidou and C. Zhang, Probing top-quark couplings indirectly at Higgs factories, Chin. Phys. C 42 (2018) 123107 [arXiv: 1809.03520] [INSPIRE].

[50] Q. Bonnefoy, L. Di Luzio, C. Grojean, A. Paul and A.N. Rossia, Comments on gauge anomalies at dimension-six in the Standard Model Effective Field Theory, JHEP 05 (2021) 153 [arXiv: 2012.07740] [INSPIRE].

[51] F. Feruglio, A Note on Gauge Anomaly Cancellation in Effective Field Theories, JHEP 03 (2021) 128 [arXiv: 2012.13989] [InSPIRE]. 
[52] A. Alloul, N.D. Christensen, C. Degrande, C. Duhr and B. Fuks, FeynRules 2.0 - A complete toolbox for tree-level phenomenology, Comput. Phys. Commun. 185 (2014) 2250 [arXiv:1310.1921] [INSPIRE].

[53] C. Degrande, C. Duhr, B. Fuks, D. Grellscheid, O. Mattelaer and T. Reiter, UFO - The Universal FeynRules Output, Comput. Phys. Commun. 183 (2012) 1201 [arXiv:1108.2040] [INSPIRE].

[54] J. Alwall et al., The automated computation of tree-level and next-to-leading order differential cross sections, and their matching to parton shower simulations, JHEP 07 (2014) 079 [arXiv: 1405.0301] [INSPIRE].

[55] T. Sjöstrand et al., An introduction to PYTHIA 8.2, Comput. Phys. Commun. 191 (2015) 159 [arXiv:1410.3012] [inSPIRE].

[56] NNPDF collaboration, Parton distributions for the LHC Run II, JHEP 04 (2015) 040 [arXiv: 1410.8849$]$ [INSPIRE].

[57] P. Artoisenet, R. Frederix, O. Mattelaer and R. Rietkerk, Automatic spin-entangled decays of heavy resonances in Monte Carlo simulations, JHEP 03 (2013) 015 [arXiv:1212.3460] [INSPIRE].

[58] J.M. Campbell, R.K. Ellis, P. Nason and E. Re, Top-Pair Production and Decay at NLO Matched with Parton Showers, JHEP 04 (2015) 114 [arXiv:1412.1828] [INSPIRE].

[59] E. Re, Single-top Wt-channel production matched with parton showers using the POWHEG method, Eur. Phys. J. C 71 (2011) 1547 [arXiv: 1009.2450] [INSPIRE].

[60] T. Melia, P. Nason, R. Rontsch and G. Zanderighi, $W+W$-, $W Z$ and $Z Z$ production in the POWHEG BOX, JHEP 11 (2011) 078 [arXiv:1107.5051] [INSPIRE].

[61] P. Nason and G. Zanderighi, $W^{+} W^{-}, W Z$ and $Z Z$ production in the POWHEG-BOX-V2, Eur. Phys. J. C $\mathbf{7 4}$ (2014) 2702 [arXiv:1311.1365] [InSPIRE].

[62] S. Alioli, P. Nason, C. Oleari and E. Re, A general framework for implementing NLO calculations in shower Monte Carlo programs: the POWHEG BOX, JHEP 06 (2010) 043 [arXiv: 1002 .2581] [INSPIRE].

[63] M. Czakon and A. Mitov, Top++: A Program for the Calculation of the Top-Pair Cross-Section at Hadron Colliders, Comput. Phys. Commun. 185 (2014) 2930 [arXiv:1112.5675] [INSPIRE].

[64] M. Czakon, P. Fiedler and A. Mitov, Total Top-Quark Pair-Production Cross Section at Hadron Colliders Through $O\left(\alpha_{S}^{4}\right)$, Phys. Rev. Lett. 110 (2013) 252004 [arXiv:1303.6254] [INSPIRE].

[65] C. Anastasiou, L.J. Dixon, K. Melnikov and F. Petriello, High precision QCD at hadron colliders: Electroweak gauge boson rapidity distributions at NNLO, Phys. Rev. D 69 (2004) 094008 [hep-ph/0312266] [INSPIRE].

[66] R. Gavin, Y. Li, F. Petriello and S. Quackenbush, W Physics at the LHC with FEWZ 2.1, Comput. Phys. Commun. 184 (2013) 208 [arXiv:1201.5896] [INSPIRE].

[67] U. Haisch and G. Polesello, Searching for dark matter in final states with two jets and missing transverse energy, JHEP 02 (2019) 128 [arXiv: 1812.08129] [INSPIRE].

[68] U. Haisch and G. Polesello, Resonant third-generation leptoquark signatures at the Large Hadron Collider, JHEP 05 (2021) 057 [arXiv: 2012.11474] [INSPIRE]. 
[69] S. Catani, F. Krauss, R. Kuhn and B.R. Webber, QCD matrix elements + parton showers, JHEP 11 (2001) 063 [hep-ph/0109231] [INSPIRE].

[70] ATLAS collaboration, Search for new phenomena in events with an energetic jet and missing transverse momentum in pp collisions at $\sqrt{s}=13 \mathrm{TeV}$ with the ATLAS detector, Phys. Rev. D 103 (2021) 112006 [arXiv:2102.10874] [InSPIRE].

[71] M. Cacciari, G.P. Salam and G. Soyez, The anti- $k_{t}$ jet clustering algorithm, JHEP 04 (2008) 063 [arXiv: 0802.1189] [INSPIRE].

[72] M. Cacciari, G.P. Salam and G. Soyez, FastJet User Manual, Eur. Phys. J. C 72 (2012) 1896 [arXiv:1111.6097] [INSPIRE].

[73] ATLAS collaboration, The ATLAS Experiment at the CERN Large Hadron Collider, 2008 JINST 3 S08003 [INSPIRE].

[74] ATLAS collaboration, Expected Performance of the ATLAS Experiment - Detector, Trigger and Physics, arXiv:0901.0512 [INSPIRE].

[75] ATLAS collaboration, ATLAS b-jet identification performance and efficiency measurement with $t \bar{t}$ events in pp collisions at $\sqrt{s}=13 \mathrm{TeV}$, Eur. Phys. J. C 79 (2019) 970 [arXiv: 1907.05120] [INSPIRE].

[76] U. Haisch and G. Polesello, Searching for heavy Higgs bosons in the $t \bar{t} Z$ and $t b W$ final states, JHEP 09 (2018) 151 [arXiv: 1807.07734] [INSPIRE].

[77] M.L. Graesser and J. Shelton, Hunting Mixed Top Squark Decays, Phys. Rev. Lett. 111 (2013) 121802 [arXiv:1212.4495] [INSPIRE].

[78] ATLAS collaboration, Search for top squark pair production in final states with one isolated lepton, jets, and missing transverse momentum in $\sqrt{s}=8 \mathrm{TeV}$ pp collisions with the ATLAS detector, JHEP 11 (2014) 118 [arXiv:1407.0583] [INSPIRE].

[79] ATLAS collaboration, Object-based missing transverse momentum significance in the ATLAS detector, Tech. Rep. ATLAS-CONF-2018-038 (2018).

[80] C.G. Lester and D.J. Summers, Measuring masses of semiinvisibly decaying particles pair produced at hadron colliders, Phys. Lett. B 463 (1999) 99 [hep-ph/9906349] [INSPIRE].

[81] ATLAS collaboration, Search for a scalar partner of the top quark in the all-hadronic t $\bar{t}$ plus missing transverse momentum final state at $\sqrt{s}=13 \mathrm{TeV}$ with the ATLAS detector, Eur. Phys. J. C 80 (2020) 737 [arXiv:2004.14060] [inSPIRE].

[82] D. Dercks, N. Desai, J.S. Kim, K. Rolbiecki, J. Tattersall and T. Weber, CheckMATE 2: From the model to the limit, Comput. Phys. Commun. 221 (2017) 383 [arXiv:1611.09856] [INSPIRE].

[83] DELPHES 3 collaboration, DELPHES 3, A modular framework for fast simulation of a generic collider experiment, JHEP 02 (2014) 057 [arXiv:1307.6346] [INSPIRE].

[84] ATLAS collaboration, Search for new phenomena in final states with an energetic jet and large missing transverse momentum in pp collisions at $\sqrt{s}=13 \mathrm{TeV}$ using the ATLAS detector, Phys. Rev. D 94 (2016) 032005 [arXiv:1604.07773] [INSPIRE].

[85] ATLAS collaboration, Search for dark matter and other new phenomena in events with an energetic jet and large missing transverse momentum using the ATLAS detector, JHEP 01 (2018) 126 [arXiv:1711.03301] [rNSPIRE]. 
[86] CMS collaboration, Search for dark matter produced with an energetic jet or a hadronically decaying $W$ or $Z$ boson at $\sqrt{s}=13 \mathrm{TeV}$, JHEP 07 (2017) 014 [arXiv:1703.01651] [INSPIRE].

[87] ATLAS collaboration, Search for new phenomena in events with jets and missing transverse momentum in pp collisions at $\sqrt{s}=13 \mathrm{TeV}$ with the ATLAS detector, Tech. Rep. ATLAS-CONF-2020-048 (2020).

[88] J.M. Lindert et al., Precise predictions for $V+$ jets dark matter backgrounds, Eur. Phys. J. C 77 (2017) 829 [arXiv:1705.04664] [INSPIRE].

[89] ATLAS collaboration, Combination of searches for invisible Higgs boson decays with the ATLAS experiment, Tech. Rep. ATLAS-CONF-2020-052 (2020).

[90] M. Cepeda et al., Report from Working Group 2: Higgs Physics at the HL-LHC and HE-LHC, CERN Yellow Rep. Monogr. 7 (2019) 221 [arXiv: 1902.00134] [INSPIRE].

[91] A.L. Read, Presentation of search results: The CL(s) technique, J. Phys. G 28 (2002) 2693 [INSPIRE].

[92] L. Moneta et al., The RooStats Project, PoS ACAT2010 (2010) 057 [arXiv:1009.1003] [INSPIRE].

[93] J. Hisano, K. Ishiwata and N. Nagata, Gluon contribution to the dark matter direct detection, Phys. Rev. D 82 (2010) 115007 [arXiv: 1007.2601] [INSPIRE].

[94] M. Freytsis and Z. Ligeti, On dark matter models with uniquely spin-dependent detection possibilities, Phys. Rev. D 83 (2011) 115009 [arXiv:1012.5317] [INSPIRE].

[95] J. Hisano, K. Ishiwata, N. Nagata and T. Takesako, Direct Detection of Electroweak-Interacting Dark Matter, JHEP 07 (2011) 005 [arXiv: 1104.0228] [INSPIRE].

[96] R.J. Hill and M.P. Solon, Universal behavior in the scattering of heavy, weakly interacting dark matter on nuclear targets, Phys. Lett. B 707 (2012) 539 [arXiv:1111.0016] [INSPIRE].

[97] M.T. Frandsen, U. Haisch, F. Kahlhoefer, P. Mertsch and K. Schmidt-Hoberg, Loop-induced dark matter direct detection signals from gamma-ray lines, JCAP 10 (2012) 033 [arXiv:1207.3971] [INSPIRE].

[98] U. Haisch and F. Kahlhoefer, On the importance of loop-induced spin-independent interactions for dark matter direct detection, JCAP 04 (2013) 050 [arXiv:1302.4454] [INSPIRE].

[99] R.J. Hill and M.P. Solon, WIMP-nucleon scattering with heavy WIMP effective theory, Phys. Rev. Lett. 112 (2014) 211602 [arXiv:1309.4092] [InSPIRE].

[100] A. Crivellin, F. D'Eramo and M. Procura, New Constraints on Dark Matter Effective Theories from Standard Model Loops, Phys. Rev. Lett. 112 (2014) 191304 [arXiv: 1402.1173] [INSPIRE].

[101] A. Crivellin and U. Haisch, Dark matter direct detection constraints from gauge bosons loops, Phys. Rev. D 90 (2014) 115011 [arXiv:1408.5046] [InSPIRE].

[102] F. D'Eramo and M. Procura, Connecting Dark Matter UV Complete Models to Direct Detection Rates via Effective Field Theory, JHEP 04 (2015) 054 [arXiv:1411.3342] [INSPIRE].

[103] F. D'Eramo, B.J. Kavanagh and P. Panci, You can hide but you have to run: direct detection with vector mediators, JHEP 08 (2016) 111 [arXiv:1605.04917] [INSPIRE]. 
[104] F. Bishara, J. Brod, B. Grinstein and J. Zupan, Renormalization Group Effects in Dark Matter Interactions, JHEP 03 (2020) 089 [arXiv: 1809.03506] [INSPIRE].

[105] P. Junnarkar and A. Walker-Loud, Scalar strange content of the nucleon from lattice QCD, Phys. Rev. D 87 (2013) 114510 [arXiv:1301.1114] [INSPIRE].

[106] M. Hoferichter, J. Ruiz de Elvira, B. Kubis and U.-G. Meißner, High-Precision Determination of the Pion-Nucleon $\sigma$ Term from Roy-Steiner Equations, Phys. Rev. Lett. 115 (2015) 092301 [arXiv: 1506. 04142] [INSPIRE].

[107] XENON collaboration, Dark Matter Search Results from a One Ton-Year Exposure of XENON1T, Phys. Rev. Lett. 121 (2018) 111302 [arXiv:1805.12562] [INSPIRE].

[108] J. McDonald, Gauge singlet scalars as cold dark matter, Phys. Rev. D 50 (1994) 3637 [hep-ph/0702143] [INSPIRE].

[109] G. Bélanger, F. Boudjema, A. Goudelis, A. Pukhov and B. Zaldivar, MicrOMEGAs5.0: Freeze-in, Comput. Phys. Commun. 231 (2018) 173 [arXiv:1801.03509] [InSPIRE].

[110] Planck collaboration, Planck 2018 results. VI. Cosmological parameters, Astron. Astrophys. 641 (2020) A6 [Erratum ibid. 652 (2021) C4] [arXiv: 1807. 06209] [InSPIRE].

[111] FERMI-LAT and DES collaborations, Searching for Dark Matter Annihilation in Recently Discovered Milky Way Satellites with Fermi-LAT, Astrophys. J. 834 (2017) 110 [arXiv: 1611.03184] [INSPIRE].

[112] FERMI-LAT collaboration, Updated search for spectral lines from Galactic dark matter interactions with pass 8 data from the Fermi Large Area Telescope, Phys. Rev. D 91 (2015) 122002 [arXiv: 1506.00013] [INSPIRE].

[113] J.F. Navarro, C.S. Frenk and S.D.M. White, The Structure of cold dark matter halos, Astrophys. J. 462 (1996) 563 [astro-ph/9508025] [INSPIRE].

[114] ATLAS collaboration, Combination of searches for invisible Higgs boson decays with the ATLAS experiment, Phys. Rev. Lett. 122 (2019) 231801 [arXiv:1904.05105] [INSPIRE].

[115] CMS collaboration, Search for invisible decays of a Higgs boson produced through vector boson fusion in proton-proton collisions at $\sqrt{s}=13 \mathrm{TeV}$, Phys. Lett. B 793 (2019) 520 [arXiv: 1809.05937] [INSPIRE].

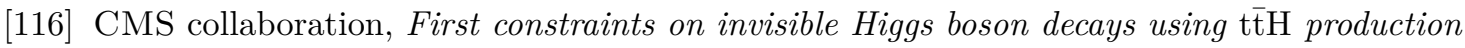
at $\sqrt{s}=13 \mathrm{TeV}$, Tech. Rep. CMS-PAS-HIG-18-008 (2019).

[117] S. Matsumoto et al., Observing the Coupling between Dark Matter and Higgs Boson at the ILC, in International Linear Collider Workshop, (2010) [arXiv: 1006.5268] [INSPIRE].

[118] S. Kanemura, S. Matsumoto, T. Nabeshima and H. Taniguchi, Testing Higgs portal dark matter via $Z$ fusion at a linear collider, Phys. Lett. B 701 (2011) 591 [arXiv:1102.5147] [INSPIRE].

[119] Z. Chacko, Y. Cui and S. Hong, Exploring a Dark Sector Through the Higgs Portal at a Lepton Collider, Phys. Lett. B 732 (2014) 75 [arXiv:1311.3306] [InSPIRE].

[120] N. Craig, H.K. Lou, M. McCullough and A. Thalapillil, The Higgs Portal Above Threshold, JHEP 02 (2016) 127 [arXiv: 1412.0258] [INSPIRE].

[121] P. Ko and H. Yokoya, Search for Higgs portal DM at the ILC, JHEP 08 (2016) 109 [arXiv: 1603. 04737] [INSPIRE]. 
[122] T. Hahn, Generating Feynman diagrams and amplitudes with FeynArts 3, Comput. Phys. Commun. 140 (2001) 418 [hep-ph/0012260] [INSPIRE].

[123] T. Hahn and M. Pérez-Victoria, Automatized one loop calculations in four-dimensions and D-dimensions, Comput. Phys. Commun. 118 (1999) 153 [hep-ph/9807565] [INSPIRE].

[124] T. Hahn, S. Paßehr and C. Schappacher, FormCalc 9 and Extensions, PoS LL2016 (2016) 068 [arXiv: 1604.04611] [INSPIRE].

[125] R. Mertig, M. Böhm and A. Denner, FEYN CALC: Computer algebraic calculation of Feynman amplitudes, Comput. Phys. Commun. 64 (1991) 345 [InSPIRE].

[126] V. Shtabovenko, R. Mertig and F. Orellana, New Developments in FeynCalc 9.0, Comput. Phys. Commun. 207 (2016) 432 [arXiv:1601.01167] [InSPIRE].

[127] V. Shtabovenko, R. Mertig and F. Orellana, FeynCalc 9.3: New features and improvements, Comput. Phys. Commun. 256 (2020) 107478 [arXiv:2001.04407] [INSPIRE]. 